\title{
HOMOTOPY TYPE THEORY AND VOEVODSKY'S UNIVALENT FOUNDATIONS
}

\author{
ÁLVARO PELAYO AND MICHAEL A. WARREN
}

Abstract. Recent discoveries have been made connecting abstract homotopy theory and the field of type theory from logic and theoretical computer science. This has given rise to a new field, which has been christened homotopy type theory. In this direction, Vladimir Voevodsky observed that it is possible to model type theory using simplicial sets and that this model satisfies an additional property, called the Univalence Axiom, which has a number of striking consequences. He has subsequently advocated a program, which he calls univalent foundations, of developing mathematics in the setting of type theory with the Univalence Axiom and possibly other additional axioms motivated by the simplicial set model. Because type theory possesses good computational properties, this program can be carried out in a computer proof assistant. In this paper we give an introduction to homotopy type theory in Voevodsky's setting, paying attention to both theoretical and practical issues. In particular, the paper serves as an introduction to both the general ideas of homotopy type theory as well as to some of the concrete details of Voevodsky's work using the well-known proof assistant Coq. The paper is written for a general audience of mathematicians with basic knowledge of algebraic topology; the paper does not assume any preliminary knowledge of type theory, logic, or computer science. Because a defining characteristic of Voevodsky's program is that the Coq code has fundamental mathematical content, and many of the mathematical concepts which are efficiently captured in the code cannot be explained in standard mathematical English without a lengthy detour through type theory, the later sections of this paper (beginning with Section 3) make use of code; however, all notions are introduced from the beginning and in a self-contained fashion.

\section{Contents}

1. Introduction

2. Origins, basic aspects, and current research

2.1. The homotopy theoretic interpretation of type theory 601

2.2. Dependent products

2.3. Inductive types

2.4. Groupoids

2.5. The univalent model of type theory

Received by the editors October 20, 2012.

2010 Mathematics Subject Classification. Primary 03-02; Secondary 03-B15, 68N18, 55P99.

The first author was partly supported by NSF CAREER Award DMS-1055897, Spain Ministry of Science Grant MTM 2010-21186-C02-01, and Spain Ministry of Science Sev-2011-0087. He also received support from NSF Grant DMS-0635607 during the preparation of this paper.

The second author received support from the Oswald Veblen Fund and NSF Grant DMS0635607 during the preparation of this paper. 
2.6. The univalent perspective

2.7. Computational aspects

2.8. Reasoning about spaces in type theory

3. Basic Coq constructions

3.1. The Coq proof assistant

3.2. Types and terms in Coq

3.3. A direct definition involving function spaces

3.4. An indirect definition involving function spaces

4. Some basic inductive types

4.1. The inductive type of natural numbers

4.2. Fibrations and the total space of a fibration

5. The path space

5.1. Groupoid structure of the path space

5.2. The functorial action of a continuous map on a path 623
6. Transport

6.1. Homotopy and homotopy equivalence 625

6.2. Forward and backward transport 626

6.3. Paths in the total space

7. Weak equivalences and homotopy equivalences

7.1. Contractibility 629

7.2. Homotopy fibers 631

7.3. Weak equivalences 635

7.4. Weak equivalences and homotopy equivalences 636

8. The Univalence Axiom and some consequences 638

8.1. The Univalence Axiom 638

8.2. An equivalent formulation of the Univalence Axiom 638

8.3. Function extensionality 640

8.4. Closure of h-levels under arbitrary products 641

8.5. The total space and h-levels 641

8.6. The unit type and contractibility 642

8.7. Some propositions 642

8.8. The h-levels of h-universes

9. Future directions

Acknowledgments

About the authors

References 645

\section{INTRODUCTION}

Type theory is a branch of mathematical logic which developed out of the work of Alonzo Church [14 16] and which has subsequently found many applications in theoretical computer science, especially in the theory of programming languages [58. For instance, the notion of datatype in programming languages derives from the type theoretic notion of type. Recently, a number of deep and unexpected connections between a form of type theory (introduced by Per Martin-Löf 29, 51 $[53$ ) and homotopy theory have been discovered, opening the way to a new research area in mathematics and theoretical computer science which has recently 
been christened homotopy type theory. Due to the nature of the mathematical results in homotopy type theory, we believe that there is great potential for future research in this field to have a considerable impact on a number of areas of pure and applied mathematics, as well as on the practice of mathematics.

In 1998, Hofmann and Streicher [29] constructed a model of Martin-Löf type theory in the category of groupoids (see Section 2.4). They also observed that the data of type theory itself naturally gives rise to a kind of $\infty$-groupoid structure (although they did not prove this for any precise definition of $\infty$-groupoid). In 2001, Moerdijk speculated that there might be some connection between Quillen model categories and type theory. Then between 2005 and 2006 Awodey and Warren 5,80 , 81] and Voevodsky [75 78] independently understood how to interpret type theory using ideas from homotopy theory (in the former case, using the general machinery of Quillen model categories and weak factorization systems, and in the latter case using simplicial sets). Subsequently, around 2009, Voevodsky 76 realized that the model of type theory in simplicial sets satisfies an additional axiom, which he called the Univalence Axiom, that does not hold in general (see Section 2.5). Crucially, satisfaction of the Univalence Axiom is a property which distinguishes Voevodsky's model of type theory in simplicial sets from the more familiar set theoretic model (it does not hold in the latter).

These results and others (described in more detail below) give rise to what might be called the univalent perspective, wherein one works in a formal system (namely, type theory augmented by additional axioms, such as the Univalence Axiom, which hold in the simplicial set model) which gives us at the outset the world of spaces (homotopy types), without having first to "build" them from the empty set and basic operations on sets. In this setting, all of the sets are still available, and it is consistent for the sets to possess their usual properties (in the simplicial set model they are exactly the familiar ZFC sets), but they are "carved out of" or extracted from the universe as discrete spaces.

Something which is not revealed in this simple comparison is that it is considerably easier to extract sets from the world of homotopy types than it is to construct homotopy types from sets. It is also easier to work with "higher-level" mathematical structures in the univalent setting than in the familiar set theoretic setting. We refer to Section 2.6 for further information in this direction.

Although interesting in its own right, this perspective becomes significantly more notable in light of the good computational properties of the kind of type theory employed here. In particular, type theory forms the underlying theoretical framework of several computer proof assistants such as Agda and Coq (see [18] and [9], respectively), and these can be used to formalize mathematics done in the univalent setting.

In practical terms, this means that it is possible to develop mathematics involving spaces in computer systems which are capable of verifying the correctness of proofs and of providing some degree of automation of proofs. We refer the reader to 69]

TABLE 1. Sets and spaces from set theoretic and univalent perspectives.

\begin{tabular}{l|cc} 
& set theoretic & univalent \\
\hline spaces & constructed & given \\
sets & given & extracted
\end{tabular}


and [27] for two accounts of computer proof assistants (and related developments) written for a general mathematical audience.

Since his aforementioned discoveries, Voevodsky has been advocating the formalization of mathematics in proof assistants, as well as greater interaction between the developers of computer proof assistants and pure mathematicians. He has himself written thousands of lines of code in the Coq proof assistant, documenting topics ranging from the development of homotopy theoretic notions and proofs of new results in type theory, to the formalization of the basics of abstract algebra (see [79] for a survey of the library written by Voevodsky himself).

Voevodsky's univalent perspective, as detailed in his Coq files, is a unique view of mathematics, and we believe that it deserves to be more widely known. Unfortunately, for a mathematician without some background in type theory, homotopy theory, and category theory, we believe that the prospect of reading thousands of lines of Coq code is likely rather daunting (indeed, it may be daunting even for those with the prerequisites listed above). In this paper we attempt to remedy this by providing an introduction to both homotopy type theory and the univalent perspective, as well as to some of the material contained in Voevodsky's Coq files. It is our hope that the reader who is not interested in the Coq code but who is curious about homotopy type theory will benefit from an account of this field specifically targeted at a general mathematical audience. For those who are interested in Coq code, we believe that this paper can act as an accessible introduction. Indeed, it is our ultimate aim that this paper will encourage other mathematicians to become involved in this area and in the use of computer proof assistants in general.

Disclaimers. This article is written for a broad audience of mathematicians who are not necessarily familiar with type theory and homotopy theory (a briefer introduction can be found in [4). Because of the introductory nature of the article, we are less precise than one would be in a research article. This is especially true when it comes to describing type theory and Coq, where we eschew excessive terminology and notation in favor of a more informal approach. For those readers with the requisite background in logic and category theory who are interested in a more detailed account, we refer to [2]. Needless to say, in the present article the authors have no intention of being comprehensive; this is merely an invitation to a new and exciting subject. For a more comprehensive introduction to homotopy type theory and with a somewhat complementary focus and perspective, see the new book 32 .

Readers who do not intend to implement proofs in Coq and who are only interested in understanding the main ideas of Voevodsky's program will be primarily interested in Sections 1 and 2 Those who, in addition, are interested in proof assistants and some of the basics of Coq should also read Sections 3 and 4 . Those more ambitious readers who want a deeper understanding or who are interested in working on this program should also read Sections 5, 6, 0, and 8. These sections introduce the concepts of homotopy type theory and Voevodsky's program, and we believe that they are better explained in the context of Coq code. Finally, Section 9 elaborates on some major open problems and research directions.

There are three main reasons we have chosen to include a significant amount of Coq code in the body of this paper. First, as we understand it, one of the core aims of Voevodsky's program is to encourage the adoption of proof assistants as a means of formally verifying mathematical proofs, while at the same time providing theoretical insights from mathematics which will lead to advances in the technology 
of proof assistants. Second, much of Voevodsky's own work in univalent foundations can be found in his Coq libraries. However, without an introduction geared towards his development, this mathematical work remains inaccessible to the average mathematician. We believe that the inclusion of Coq code here will make Voevodsky's work in this area accessible to those who are interested. Finally, the Coq code has fundamental mathematical content, and many of the mathematical concepts which are efficiently captured in the code cannot be explained in standard mathematical English without a lengthy detour through type theory. While such a detour is certainly worth making for the interested mathematician, we believe that its inclusion here would be unwarranted.

There are already a number of introductions to Coq available (see for instance [9]) which are far more comprehensive and precise than this article in their treatment of the proof assistant itself. However, such introductions inevitably make use of features of the Coq system which do not enter into (or are even incompatible with) the univalent perspective. As such, we warn the reader that this paper is not a Coq tutorial: it is an introduction to the univalent perspective which along the way also describes some of the basic features of Coq.

Finally, Coq is not an automatic theorem prover, but rather an interactive theorem prover: it helps one to verify the correctness of proofs which are themselves provided by the user 1

\section{ORIGins, BASIC ASPECTS, AND CURRENT RESEARCH}

This section gives an overview of the origins of homotopy type theory and Voevodsky's univalent perspective. We will introduce type theory-already with the homotopy theoretic interpretation of [5, 76] in mind-by analogy with certain developments and constructions in algebraic topology. This approach is admittedly anachronistic, but we hope that it will serve as an accessible starting point for readers coming from outside of type theory. Along the way we will try to give an idea of the historical development of the field. However, we make no attempt to provide a comprehensive history of homotopy theory or type theory. For the early history of homotopy theory we refer to 21 .

2.1. The homotopy theoretic interpretation of type theory. Although the mathematical notion of type first appears in Russell's work 61 on the foundations of mathematics, it was not until the work of Church 15 that type theory in its modern form was born. Later, building on work by Curry 20, Howard 31, Tait [72, Lawvere [39], de Bruijn [11, Scott 62], and others, Martin-Löf [29, 51,53. developed a generalization of Church's system, which is now called dependent or Martin-Löf type theory. We will be exclusively concerned with this form of type theory and so the term "type theory" henceforth refers to this particular system 2

\footnotetext{
${ }^{1}$ Technically, it is possible to automate proofs to a large extent in Coq (via the built-in tactics language), but, aside from a minor amount of automation implicit in the "tactics", we employ below, we will not go into details regarding these features of Coq. The interested reader might consult [13] for a good introduction to Coq which pays particular attention to automation.

${ }^{2}$ We caution the reader that there are indeed many different kinds of type theory. Explicitly, we are concerned with the intensional form of dependent type theory. One can ask, "But what is a type theory?" This is an interesting question. However, just as the question "What is a set theory?" is eschewed in most (if not all) surveys of set theory (there are also many different kinds of set theory), we will not consider this question here.
} 
Type theory, like set theory, is a formal theory which is given by a collection of rules. Anyone interested in type theory should at some point study these rules, but doing so is not strictly necessary in order to give some flavor of the theory. As such, we choose to abstain from giving a fully formal presentation of type theory. There is however a difference between set theory and type theory at this formal level. Formulating set theory precisely requires all of the deductive machinery of (first-order) logic in addition to the axioms describing the behavior of sets. In this way, set theory (like, say, the theory of rings) is built on top of logic. In type theory by contrast all of the deductive machinery is built in to the theory itself. Consequently, the formal syntax of type theory may appear at first glance more complicated than the formal syntax of set theory. This apparent complexity is not without reason: it gives rise to many of the good properties and applications of type theory.

Type theory is concerned principally with expressions of the form

$$
a: A
$$

which asserts that the term $a$ is of type $A$. Here we already see a difference with set theory: in set theory all that we can speak about are sets, whereas type theory is concerned with both types and terms.

There are a number of ways that $a: A$ has traditionally been motivated:

(1) $A$ is a set and $a$ is an element of $A$.

(2) $A$ is a problem and $a$ is a solution of $A$.

(3) $A$ is a proposition and $a$ is a proof of $A$.

Roughly, of the perspectives enumerated here, the first is due to Russell [61, the second is due to Kolmogorov 38, and the third-usually called the Curry-Howard correspondence - is due to Curry and Howard [31. We will say more about these three motivations in the sequel. Although (1)-(3) are often given as a heuristic way of motivating the notions of types and terms, they can be made mathematically precise. (1) is the most pertinent for us.

The starting point for understanding the connections between homotopy theory and type theory is to consider a fourth alternative to these motivations:

(4) $A$ is a space and $a$ is a point of $A$.

That is, (4) is the generalization of (1) to spaces which are not necessarily discrete. We will be intentionally vague about exactly what kinds of spaces we are considering, but we recommend that readers have in mind, e.g., topological spaces (better yet) CW-complexes, or (still better yet) Kan complexes (in which case, "point" means 0 -simplex). The remarkable thing about (4) is that it helps to clarify certain features of type theory which originally seemed odd, or even undesirable, from the point of view of interpretations (1)-(3) above.

In addition to the kinds of types and terms described above, we also may consider types and terms with parameters. These are usually called dependent types and terms. E.g., when $B$ is a type, we write

$$
x: B \vdash E(x),
$$

to indicate that we have a type $E(x)$ which is parameterized by $B$ (here $x$ is a variable). In terms of the motivation (1) above in terms of sets, we would think of this as a $B$-indexed family $\left(E_{x}\right)_{x \in B}$. In terms of $(3)$, we think of this as a predicate on $B$, i.e., a property of terms of type $B$. From the homotopy theoretic 


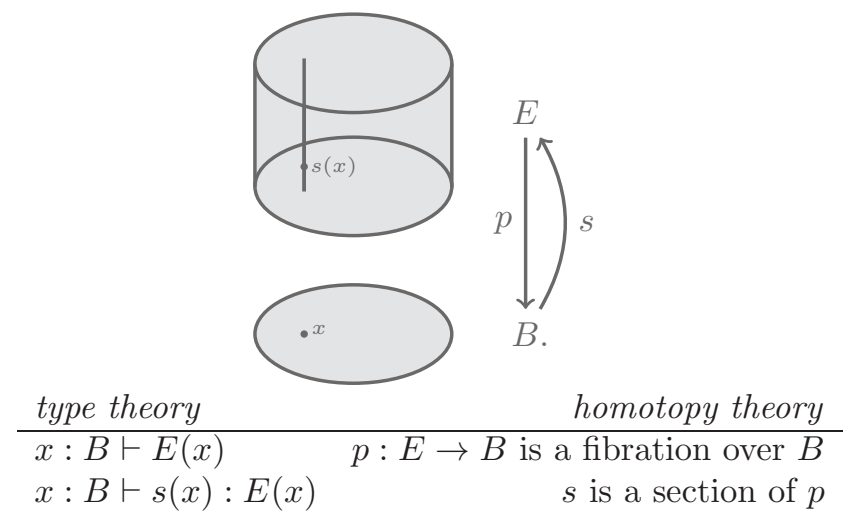

FiguRE 1. Homotopy theoretic interpretation of dependent types and terms.

point of view (4), we think of such a type as describing a fibration $E \rightarrow B$ over the space $B$. Technically, fibrations are maps satisfying a certain homotopy lifting property; informally, they may be thought of as families of spaces $\left(E_{x}\right)_{x \in B}$ which "fit together" in accordance with the homotopy theoretic structure of the base space $B$.

Similarly, we think of a parameterized term

$$
x: B \vdash s(x): E(x)
$$

as a continuous section of the fibration $E \rightarrow B$. Types and terms are allowed to depend on finitely many other types. I.e., we may have

$$
x_{1}: A_{1}, x_{2}: A_{2}\left(x_{1}\right), \ldots, x_{n}: A_{n}\left(x_{1}, \ldots, x_{n-1}\right) \vdash E\left(x_{1}, \ldots, x_{n}\right) .
$$

Of course, none of this would be useful without being given some types and terms to start with and some rules for generating new types and terms from old ones. It is to these that we now turn.

2.2. Dependent products. Given a dependent type $x: B \vdash E(x)$, there exists a type $\prod_{x: B} E(x)$ called the dependent product of $x: B \vdash E(x)$. Intuitively, $\prod_{x: B} E(x)$ is a kind of parameterized function space. In the set theoretic interpretation this is the usual set-theoretic product $\prod_{x \in B} E_{x}$ of the family of sets $\left(E_{x}\right)_{x \in B}$. From the homotopical point of view, this operation takes a fibration $E \rightarrow B$ and gives back the space of all continuous sections of the fibration. That is, we should think of a point $s: \prod_{x: B} E(x)$ of this type as corresponding to a continuous section

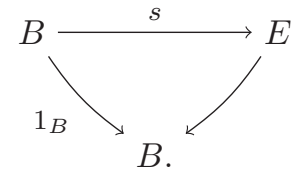

The rules for dependent products are consistent with this interpretation. In particular, we have a rule which states that if we are given a term (section) $x: B \vdash s(x): E(x)$, then there is a corresponding term $\lambda_{x: B} s(x): \prod_{x: B} E(x)$. This rule is often expressed as 


$$
\frac{x: B \vdash s(x): E(x)}{\lambda_{x: B} s(x): \prod_{x: B} E(x)},
$$

which indicates that whenever data of the form above the line is provided, then we have the term indicated below the line. Here the variable $x$ is bound in the term $\lambda_{x: B} s(x)$ and in the type $\prod_{x: B} E(x)$. I.e., a continuous section of $E \rightarrow B$ determines a point in the space of all such continuous sections.

The next rule shows us how to use terms of dependent product type. Given terms $f: \prod_{x: B} E(x)$ and $b: B$, we obtain a further term $\operatorname{app}(f, b): E(b)$. Written in the form introduced above, this rule can be stated as

$$
\frac{f: \prod_{x: B} E(x) \quad b: B}{\operatorname{app}(f, b): E(b)} .
$$

In the interpretation the term $\operatorname{app}(f, b)$ corresponds to the result $f(b)$ of applying the function $f$ to the point $b$.

The final rule governing dependent products describes the interaction between application and the " $\lambda$-abstraction" $\lambda_{x: B} s(x)$ :

$$
\frac{x: B \vdash s(x): E(x) \quad b: B}{\operatorname{app}\left(\lambda_{x: B} s(x), b\right)=s(b): E(b)} .
$$

The dependent product can be used to construct function spaces. Given two types $A$ and $B$, the "function space" type $B \rightarrow A$ is constructed by regarding (trivially) the type $A$ as being parameterized by $B$ (i.e., $x: B \vdash A$, which corresponds to the trivial fibration $\pi_{1}: B \times A \rightarrow B$ ). Then we define $B \rightarrow A$ to be the dependent product $\prod_{x: B} A$. We will now turn to some of the further ways of constructing types.

2.3. Inductive types. Among all of the types which can be constructed, some of the most significant and interesting are the inductive types. In non-technical terms, inductive types are types which are freely generated in an appropriate (type theoretic) sense. Before describing in more detail the notion of inductive type, we will first consider a simple example.

The most familiar example of an inductive type is the type nat of natural numbers. As a type, nat is freely generated by the following data:

- a term 0: nat (the natural number zero); and

- a term $x$ : nat $\vdash \mathrm{S}(x)$ : nat (the successor function).

To say that nat is freely generated by this data is to say that it is the smallest space possessing a term and an endomorphism subject to no further conditions. To say that it is the smallest is important. For example, the real numbers are not freely generated by their successor endomorphism and zero. To say that the generating endomorphism is subject to no further conditions is also important. For example, the one point type $\{*\}$ possesses an element $*$ and an endomorphism (the identity) and is smaller than the natural numbers. However, by making the endomorphism the identity function, we have imposed additional constraints on this structure. Therefore, $\{*\}$ cannot be said to be freely generated in the same sense. The reader who is puzzled by these points should consider by analogy the case of finitely presented groups: a group may fail to be the group generated by a specified finite presentation by virtue of possessing elements not obtainable from the generators or by virtue of satisfying additional equations not derivable from the group laws and the relations. 
This is achieved by the following type theoretic rule, which should be familiar as proof by induction (alternatively, definition by recursion):

$$
\frac{x: \text { nat } \vdash E(x) \quad e: E(0) \quad x: \text { nat, } y: E(x) \vdash f(x, y): E(\mathrm{~S}(x))}{x: \text { nat } \vdash \operatorname{rec}(e, f, x): E(x) .}
$$

This rule tells us that, given any type

$$
x: \text { nat } \vdash E(x)
$$

fibered over nat, in order to construct a term of type $x:$ nat $\vdash E(x)$, it suffices to provide terms

- $e: E(0)$ (base case); and

- $x$ : nat, $y: E(x) \vdash f(x, y): E(\mathrm{~S}(x))$ (induction step).

The remaining rules for nat require that the following equations hold:

$$
\begin{aligned}
\operatorname{rec}(e, f, 0) & =e \quad \text { and } \\
\operatorname{rec}(e, f, \mathrm{~S}(x)) & =f(x, \operatorname{rec}(e, f, x)) .
\end{aligned}
$$

This should be compared with the usual way that functions from the natural numbers are defined by recursion. For example, fixing a natural number $m$, let us define the function $\alpha_{m}$ : nat $\rightarrow$ nat which acts by $n \mapsto n+m$, for $n$ : nat. First, we observe that the type nat trivially is a dependent type over itself so that we may take $x$ : nat $\vdash E(x)$ to be $x$ : nat $\vdash$ nat. The base case and induction steps are then given as

- $m$ : nat (base case) and

- $x$ : nat, $y$ : nat $\vdash \mathrm{S}(y)$ : nat (induction step).

This gives, by applying the rules above to our base and induction cases, the recursion term $x$ : nat $\vdash \operatorname{rec}(m, \mathrm{~S}(y), x)$ : nat. The map $\alpha_{m}$ : nat $\rightarrow$ nat is then defined to be the following lambda abstraction (see Section 2.2 above):

$$
\alpha_{m}:=\lambda_{x: \text { nat }} \operatorname{rec}(m, \mathrm{~S}(y), x): \text { nat } \rightarrow \text { nat. }
$$

This map then satisfies the recursion equations

$$
\begin{aligned}
\operatorname{app}\left(\alpha_{m}, 0\right) & =m \quad \text { and } \\
\operatorname{app}\left(\alpha_{m}, \mathrm{~S}(n)\right) & =\mathrm{S}\left(\operatorname{app}\left(\alpha_{m}, n\right)\right)
\end{aligned}
$$

for $n$ : nat.

It is easy to show that in the homotopy theoretic interpretation a space nat satisfies the aforementioned rules if and only if it is equivalent to the discrete space of natural numbers.

In general, it is possible to construct inductive types which are generated (in the sense that nat is freely generated by zero and successor) by arbitrary generators (subject to some technical conditions on the kinds of generators allowed). It is also possible to construct inductive types which are themselves dependent. Given a base type $B$, such a dependent inductive type $x: B \vdash I(x)$ over $B$ should be understood homotopy theoretically as the smallest fibration $I \rightarrow B$ over $B$ equipped with the specified generators and subject to no further conditions. The requirement that $I \rightarrow B$ should be a fibration will in general force the total space $I$ to possess additional structure in the same way that a finitely presented group will in general possess additional elements and to satisfy additional equations, which are not specified as part of the presentation but which are consequences of the 
group laws. We will now turn to what is one of the most significant examples of a dependent inductive type.

If $A$ is a type, consider the inductive type fibered over $A \times A$ with a single generator $\mathrm{r}(a)$ in the fiber over $(a, a)$. That is, consider the smallest (in an appropriate sense) fibration over $A \times A$ having such elements $r(a)$ in the fibers subject to no further conditions. The resulting inductive type, called the identity type of $A$, is usually written type theoretically as

$$
x: A, y: A \vdash \operatorname{Id}_{A}(x, y)
$$

with its generators written as

$$
x: A \vdash \mathrm{r}(x): \operatorname{Id}_{A}(x, x) .
$$

Type theoretically, stating that this type is inductively generated in this way is to say that the rule

$$
\frac{x: A, y: A, v: \operatorname{Id}_{A}(x, y) \vdash E(x, y, v) \quad x: A \vdash e(x): E(x, x, \mathrm{r}(x))}{x: A, y: A, v: \operatorname{Id}_{A}(x, y) \vdash \operatorname{rec}(e, x, y, v): E(x, y, v)}
$$

holds and the corresponding rec terms satisfy the equation

$$
\operatorname{rec}(e, a, a, r(a))=e(a) .
$$

Somewhat miraculously, this inductive type turns out, in the homotopical interpretation, to be a well known space: the space of all (continuous) paths in $A$.

Theorem 2.1 (Awodey and Warren [5]). The path space fibration $A^{I} \rightarrow A \times A$ has the universal property of the inductive type described above.

First, to say that $A^{I} \rightarrow A \times A$ represents a type at all is to say that this map is a fibration. To say that it has the generators described above is to say that there exists a map $r$ making

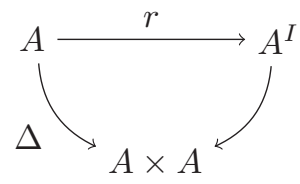

commute, where $\Delta: A \rightarrow A \times A$ is the diagonal. Here $r$ is the map which sends an element $a$ of $A$ to the constant loop $r(a)$ on $a$. Finally, the remaining rules (the existence of the $\operatorname{rec}(e, x, y, v)$ and the corresponding equation) correspond to the fact that whenever we are given a fibration $E \rightarrow A^{I}$ and a map $e$ making

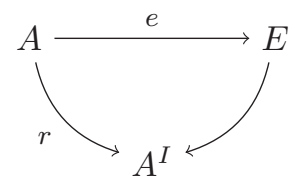

commute, there exists a map $J: A^{I} \rightarrow E$ such that

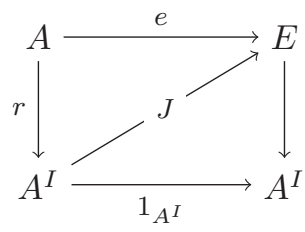


commutes. I.e., we take $x: A, y: A, v: \operatorname{Id}_{A}(x, y) \vdash \operatorname{rec}(e, x, y, v): E(x, y, v)$ to be the section $J$.

We will now consider several points regarding identity types:

(1) For non-discrete spaces $A$, there exist elements of the path space fibration not of the form $r(a)$ and arising from non-constant paths in $A$.

(2) For discrete spaces, on the other hand, the path space fibration is equivalent to the diagonal $\Delta: A \rightarrow A \times A$, meaning every element is of the form $r(a)$. (The diagonal is not in general a fibration.)

The interpretation of types as sets (discrete spaces) mentioned in Section 2.1 above is consistent with the rules we have so far described. Therefore, in order for type theory to be able to express non-discrete spaces (which would preclude the set theoretic interpretation), it is necessary to adopt further rules which reflect the behavior of these spaces. One such rule is Voevodsky's Univalence Axiom, described in Section 2.5 below. The other rules that give rise to non-constant paths govern what are called higher inductive types. Higher inductive types are a type theoretic way of describing spaces combinatorially (cf. CW-complexes, simplicial complexes, etc.) and are considered in more detail in Section 2.8 below. These rules, as well as the Univalence Axiom, give rise to non-constant paths. Once these rules have been added and we have at our disposal some non-constant paths, the rules governing identity types then give rise to further non-constant paths. Indeed, the identity types induce on types the structure of $\infty$-groupoids, which we will consider in Section 2.4 below.

Finally, Theorem 2.1 is just a small part of a more general result from [5] (see also [81] for further details): it is possible to model type theory in weak factorization systems or Quillen model categories 60] which satisfy certain coherence conditions. Going the other way, Gambino and Garner [24] showed that it is possible to construct a weak factorization system from the syntax of type theory.

2.4. Groupoids. Type theorists traditionally thought of the type $\operatorname{Id}_{A}(a, b)$ as something like the "proposition that $a$ and $b$ are equal proofs of $A$ ". It was also known that it is possible to construct a set theoretic model of type theory by thinking, as in perspective (1) above, of types as sets and terms as elements. In this set theoretic interpretation we have that

$$
\operatorname{Id}_{A}(a, b)= \begin{cases}1 & \text { if } a=b, \text { and } \\ 0 & \text { otherwise }\end{cases}
$$

I.e., $\operatorname{Id}_{A}(a, b)$ is either the one point set or the empty set depending on whether or not $a$ and $b$ are in fact equal. Under this interpretation it follows that if there is a term $f: \operatorname{Id}_{A}(a, b)$, then in fact $a=b$. This was originally seen by many, no doubt based on intuitions gleaned from the set theoretic model, as a desirable property and this property was added by many type theorists (including, for a time, Martin-Löf himself) as an axiom, which we call the truncation rule.

Type theory with the truncation rule may be somewhat easier to work with and, by work of Seely 63 and a coherence result due to Hofmann [28, it is possible, using the machinery of locally cartesian closed categories, to obtain many different models of type theory which satisfy the truncation rule. Nonetheless, adding the truncation rule destroys the good computational properties of type theory (see Section 2.7 for a brief description of the computational aspects of type theory). Many of the 
early questions in Martin-Löf type theory concerned the behavior of the identity types in the theory without truncation. In particular, much research at the time was concerned with trying to understand whether facts which are consequences of truncation also hold without this rule. Streicher's Habilitationsschrift [7] contains many fundamental results in this direction.

One important example of the kind of question about identity types that type theorists were trying to answer is the problem of "uniqueness of identity proofs": if $f$ and $g$ are both of type $\operatorname{Id}_{A}(a, b)$, does it follow that $f=g$ (or even that there exists a term $\left.\eta: \operatorname{Id}_{\operatorname{Id}_{A}(a, b)}(f, g)\right)$ ? In order to solve this problem Hofmann and Streicher 29] constructed a model of type theory in which types are interpreted as groupoids and fibrations of groupoids, and terms are interpreted as sections (see below for the notion of groupoids). In the process of constructing this model, Hofmann and Streicher discovered an interesting fact which we will now describe.

Given terms $a$ and $b$ of type $A$, there is an equivalence relation $\simeq$ on the set of terms of type $\operatorname{Id}_{A}(a, b)$ given by setting $f \simeq g$ if and only if there exists a term $\eta: \operatorname{Id}_{\operatorname{Id}_{A}(a, b)}(f, g)$. In terms of spaces, $f$ and $g$ correspond to paths from $a$ to $b$ in the space $A$, and $\eta$ corresponds to a homotopy rel endpoints from $f$ to $g$. Hofmann and Streicher realized that the quotient of the set of terms $f: \operatorname{Id}_{A}(a, a)$ modulo $\simeq$ forms a group. In fact, they realized that the type $A$ can be made into a groupoid. Recall that a groupoid is a category in which every arrow is invertible. To turn $A$ into a groupoid, we take the objects to be the terms $a: A$ and the edges to be equivalence classes of $f: \operatorname{Id}_{A}(a, b)$ modulo $\simeq$. We now see that these two constructions correspond, under the homotopy theoretic interpretation of type theory sketched above, to the constructions of the fundamental group $\pi_{1}(A, a)$ of the space $A$ with basepoint $a$ and the fundamental groupoid $\Pi_{1}(A)$ (see 10 for more on the fundamental groupoid of a space). In fact, Hofmann and Streicher realized that their construction seemed to give some kind of $\infty$-groupoid, but they did not pursue this possibility (see below for more on the notion of $\infty$-groupoid). The first non-syntactic higher-dimensional models (which are shown to satisfy all of the required coherence conditions) of type theory appeared later in work of Voevodsky [36, 76, and Warren 81,82.

In homotopy theory one is also concerned with groupoids and $\infty$-groupoids. The fundamental groupoid $\Pi_{1}(A)$ of a space $A$ is the basepoint-free generalization of Poincaré's fundamental group [59] and captures the homotopy 1-types. The objects of $\Pi_{1}(A)$ are the points of the space $A$ and the arrows from $a$ to $b$ are homotopy classes (rel endpoints) of paths from $a$ to $b$. Here a homotopy n-type is intuitively a space $A$ for which the higher-homotopy groups $\pi_{i}(A, a)$, for $i>n$, vanish. For spaces $A$ that are not 1-types, one must consider higher-dimensional generalizations of the notion of groupoid in order to capture the homotopy theoretic content of $A$. In his letter to Quillen, Grothendieck 26] emphasized the importance of finding an infinite-dimensional generalization of the notion of groupoid which would capture the homotopy types of spaces, and indeed he offered a suggestion himself (see 49] for a modern exposition of Grothendieck's definition). Such an infinite-dimensional generalization is what is commonly referred to as a notion of $\infty$ groupoid. Intuitively, an $\infty$-groupoid should be like a groupoid which possesses, in addition to objects (sometimes called 0-cells) and arrows (1-cells), arrows between arrows (2-cells), arrows between 2-cells (3-cells), and so forth (i.e., $(n+1)$-cells, for each natural number $n$, which are thought of as arrows between $n$-cells). It should 
be possible to compose these higher-dimensional cells, and there should be unit and inverse higher-dimensional cells. The typical example of such an $\infty$-groupoid should then be the fundamental $\infty$-groupoid $\Pi_{\infty}(A)$ of a space $A$ with objects the points of $A$, arrows the paths, 2-cells homotopies (rel endpoints) of paths, and so forth. As this motivating example suggests, it is unreasonable to expect the usual algebraic laws governing groupoids to hold (since, e.g., composition of arrows will now only be unital up to the existence of a 2-cell). Instead, these laws will hold up to the existence of further higher-dimensional "coherence cells" which themselves will satisfy certain laws (up to the existence of further higher-dimensional coherence cells!) The resulting complexity associated with the shift from one-dimensional algebraic structures (groupoids) in which the algebraic laws hold "on-the-nose" (up to $=$ ) to higher-dimensional algebraic structures ( $\infty$-groupoids), where these laws hold up to higher-dimensional cells, has led to numerous candidate definitions of $\infty$ groupoid in the literature, and the issue of finding a completely satisfactory notion of $\infty$-groupoid is not entirely settled. Indeed, of the different definitions of the $\infty$-groupoid in the literature only some have been shown to model homotopy types in the sense proposed by Grothendieck, and the relations between the different notions are also not completely settled. These problems have been among the leading motivations for the development of higher-dimensional category theory. We refer the reader to [7] for an overview of the problem of modeling homotopy types, to [40] for an accessible introduction to higher-dimensional category theory, to [17, 35, 42, 55, 68, 73] for some results on modeling homotopy types, and to [3, 30] for some results on type theoretic models of homotopy types.

In the setting of type theory, with respect to a Batanin-Leinster [6, 40, notion of $\infty$-groupoid following the work of Cheng [12, each type $A$ has been shown to give rise to an $\infty$-groupoid using its higher-dimensional identity types $\operatorname{Id}_{A}(a, b)$, $\operatorname{Id}_{\operatorname{Id}_{A}(a, b)}(f, g), \operatorname{Id}_{\operatorname{Id}_{\operatorname{Id}_{A}(a, b)}(f, g)}(\alpha, \beta)$, and so forth:

Theorem 2.2 (van den Berg and Garner [8], and Lumsdaine [44]). Every type A has an associated fundamental $\infty$-groupoid $\Pi_{\infty}(A)$.

Hence the set theoretic intuition for the meaning of types fails to accurately capture certain features of the type theory, whereas those features (non-trivial higherdimensional structure) are captured by the homotopy theoretic interpretation of types.

2.5. The univalent model of type theory. We mentioned above the problem of finding a notion of $\infty$-groupoid which completely captures the notion of homotopy type. One such notion is provided by Kan complexes. Introduced by Kan 34, these were shown by Quillen 60] to provide a model of homotopy types. Kan complexes are simplicial sets for which a certain combinatorial condition holds. In the work of Joyal [33 and Lurie [47] on $\infty$-toposes, $\infty$-groupoids are taken to be Kan complexes ( $\infty$-toposes in this context are simplicial sets which possess certain additional properties; a more detailed definition is beyond the scope of the present paper). The starting place for Voevodsky's univalent perspective is the following result:

Theorem 2.3 (Voevodsky [76]). Assuming the existence of Grothendieck universes (sufficiently large cardinals), there is a model of type theory in the category of simplicial sets in which types are interpreted as Kan complexes and Kan fibrations. 
We will henceforth refer to Voevodsky's model as the univalent model of type theory. The particular kind of type theory considered by Voevodsky includes a type $\mathcal{U}$ which is a universe of types that we refer to as small types 3 Given small types $A$ and $B$, it is then natural to ask what kinds of terms arise in the identity type $\operatorname{Id}_{\mathcal{U}}(A, B)$. Voevodsky realized that, although this type a priori possesses no interesting structure, in the univalent model it turns out to be non-trivial. Based on this observation, Voevodsky proposed to add to the axioms of type theory an additional axiom, called the Univalence Axiom, which would ensure that the identity type of $\mathcal{U}$ behaves as it does in the univalent model. We will now explain this axiom and some of its consequences.

It will be instructive to compare several different ways of understanding the Univalence Axiom. However, we will first start by giving an explicit description of the axiom. Given small types $A$ and $B$ there is, in addition to the identity type $\operatorname{Id}_{\mathcal{U}}(A, B)$, a type $\operatorname{WEq}(A, B)$ of weak equivalences from $A$ to $B$. Intuitively, thinking of $A$ and $B$ as spaces, a weak equivalence $f: A \rightarrow B$ is a continuous function which induces isomorphisms on homotopy groups:

$$
\pi_{n}(f): \pi_{n}(A, a) \cong \pi_{n}(B, f(a))
$$

for $n \geq 0$. The notion of weak equivalence should be contrasted with the notion of homotopy equivalence. A map $f: A \rightarrow B$ is a homotopy equivalence when there exists a map $f^{\prime}: B \rightarrow A$ together with homotopies $f^{\prime} \circ f \simeq 1_{A}$ and $f \circ f^{\prime} \simeq 1_{B}$. For sets (discrete spaces), homotopy equivalences correspond to invertible maps whereas weak equivalences correspond to bijective maps. For groupoids (homotopy 1-types) homotopy equivalences correspond to categorical equivalences of groupoids, whereas weak equivalences correspond to functors which are full, faithful, and essentially surjective on objects.

The most basic example of a weak equivalence is the identity map $1_{A}: A \rightarrow A$ on a space $A$. For $A$ and $B$ spaces in $\mathcal{U}$, this gives, by the induction principle for identity types, a map $\iota: \operatorname{Id}_{\mathcal{U}}(A, B) \rightarrow \operatorname{WEq}(A, B)$. The Univalence Axiom can be stated as follows:

Univalence Axiom (Voevodsky): For spaces $A$ and $B$ in $\mathcal{U}$, the map $\iota: \operatorname{Id}_{\mathcal{U}}(A, B) \rightarrow \operatorname{WEq}(A, B)$ is a weak equivalence.

That is, the Univalence Axiom imposes the condition that the identity type between two types is naturally weakly equivalent to the type of weak equivalences between these types. The Univalence Axiom makes it possible to automatically transport constructions and proofs between types which are connected by appropriately defined weak equivalences.

Without the Univalence Axiom there are three a priori different ways in which two small types $A$ and $B$ can be said to be equivalent:

(1) $A=B$.

(2) There exists a term $f: \operatorname{Id}_{\mathcal{U}}(A, B)$.

(3) There exists a term $f: \operatorname{WEq}(A, B)$.

The Univalence Axiom should be understood as asserting (in a type theoretic way) that (2) and (3) coincide. That is, the Univalence Axiom answers the question

\footnotetext{
${ }^{3}$ The universes in Voevodsky's model play two roles: The first is simply to interpret universes of types. The second is to avoid certain coherence issues which arise in the homotopy theoretic interpretation of type theory.
} 
"What is a path from $A$ to $B$ in the space of small spaces?" by stipulating that such a path corresponds to a weak equivalence $A \rightarrow B$.

Alternatively, the Univalence Axiom may be understood as stating that the types of the form $\operatorname{WEq}(A, B)$ are inductively generated by the identity maps $1_{A}$ : $\operatorname{WEq}(A, A)$. Part of the appeal of the Univalence Axiom is that it has a number of interesting consequences which we will discuss below. The connection between the Univalence Axiom and object classifiers from topos theory has recently been investigated by Moerdijk [54].

2.6. The univalent perspective. Following Voevodsky, we define a filtration of types by what are called $h$-levels extending the usual filtration of spaces by homotopy $n$-types. The h-levels are defined as follows:

- A type $A$ is of h-level 0 if it is contractible.

- A type $A$ is of h-level $(n+1)$ if, for all terms $a$ and $b$ of type $A$, the type $\operatorname{Id}_{A}(a, b)$ is of h-level $n$.

In order to see what this definition is saying, let us first look at spaces of h-level 1. If $A$ is a space of h-level 1 , then by definition we have that $\operatorname{Id}_{A}(a, b)$ is contractible for any points $a$ and $b$ of $A$. First, note that this condition is trivially satisfied if $A$ is the empty space. On the other hand, if $A$ is non-empty, then this condition implies that $A$ is itself contractible. Therefore, we conclude that the spaces of h-level 1 are (up to weak equivalence) the empty space and the contractible space. Accordingly, we denote by hProp the type of all (small) types of h-level 1 . The type hProp plays the same role, from the univalent perspective, as the boolean algebra $2:=\{0,1\}$ in classical logic and set theory, or the subobject classifier $\Omega$ in topos theory. We will usually refer to types in hProp as propositions. For hProp, the Univalence Axiom states that paths in hProp correspond to logical equivalences. I.e., for propositions $P$ and $Q$, it is a necessary and sufficient condition for there to exist a term of type $\operatorname{Id}_{\mathrm{hProp}}(P, Q)$ that $P$ and $Q$ should be logically equivalent.

Similarly, we denote by hSet the type of all (small) types of h-level 2 , and we refer to these types as sets. To see that this is justified, suppose $A$ is a type of hlevel 2. Then, for any terms $a$ and $b$ of type $A$, the identity type $\operatorname{Id}_{A}(a, b)$ is either empty or contractible, which is to say that $A$ is weakly equivalent to a discrete space.

For $n>1, A$ is of h-level $n$ if and only if it is a homotopy $(n-2)$-type. E.g., types of h-level 3 are the same as homotopy 1-types: spaces which are homotopy equivalent to groupoids. From a more category theoretic point of view, we can view the h-levels as shown in Table 2.

It is worth recording several basic observations. First, weak equivalences respect h-level: if there is a weak equivalence $f: A \rightarrow B$, then $A$ is of h-level $n$ if and only if $B$ is of h-level $n$. Secondly, h-levels are cumulative in the sense that if $A$ is of h-level $n$, then it is also of h-level $(n+1)$. Finally, for any $n$, if $A$ (or $B$ ) is of h-level $(n+1)$, then so is $\operatorname{WEq}(A, B)$.

For example, these observations can be used to show that hProp is a set. Note that, by the "propositional" form of the Univalence Axiom mentioned above, there is a weak equivalence $\iota: \operatorname{Id}_{\mathrm{hProp}}(A, B) \rightarrow \mathrm{WEq}(A, B)$ for propositions $A$ and $B$. It then follows from the basic observations on h-levels summarized above that $\operatorname{Id}_{\mathrm{hProp}}(A, B)$ has h-level 1 , as required. This result is a special case of the more general fact that the Univalence Axiom implies that the type hlevel ${ }_{n}$ of all (small) types of h-level $n$ is itself of h-level $(n+1)$. 
TABLE 2. h-levels.

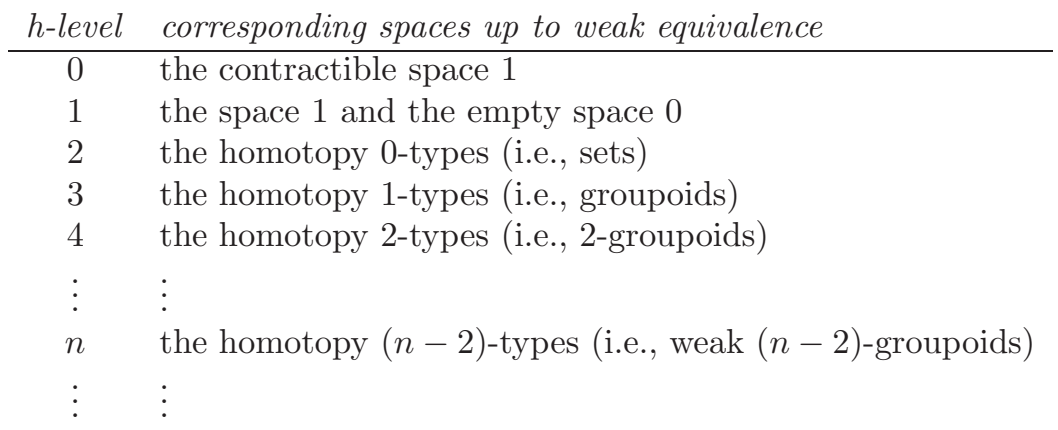

As we see it, the principal idea underlying the univalent perspective is that, rather than developing mathematics in the setting of set theory where one must "build" all of mathematics up from the emptyset and the operations of set theory, we should instead work in a formal system (namely, type theory) where we are given at the outset the world of spaces (homotopy types). In this setting we would still have all of the sets available to us, but they are "carved out of" or extracted from the universe as the types of h-level 2 .

Something which is not revealed in this simple comparison is that it is considerably easier to extract sets from the world of homotopy types than it is to construct homotopy types from sets.

From the univalent perspective, the development of ordinary "set-level mathematics", which deals with sets and structures (e.g., groups, rings, ...) on sets, is quite similar to the ordinary development of mathematics. However, in this setting it is also easy to develop "higher-level mathematics". One simple example is that the notion of monoid can be axiomatized in the ordinary way. I.e., a monoid consists of a type $M$ together with a binary operation $\mu: M \times M \rightarrow M$ which is associative and unital (in the appropriate type theoretic sense). When we restrict $M$ to just small types in hSet, we obtain the usual notion of monoid. However, when $M$ is allowed to be an arbitrary type, we obtain the notion of homotopy associative $H$-space in the sense of Serre [64. We believe that it is an advantage of the univalent perspective that it is in fact easier to work with such higher-level structures in the univalent setting than in the familiar set theoretic setting.

In addition to the fact that it is efficient to reason about higher-dimensional structures in the univalent setting, there are also technical advantages to doing so. For example, in the presence of the Univalence Axiom, any structure on a type $A$ which is type theoretically definable can be transferred along a weak equivalence $A \rightarrow B$ to give a corresponding structure on $B$. In general, being able to transfer structures along weak equivalences (or even homotopy equivalences) is non-trivial (see, e.g., 43] for some examples of such "homotopy transfer theorems" and their consequences). Therefore, being able to work in a setting where such transfer is "automatic" is technically quite convenient.

2.7. Computational aspects. One of the advantages of working with the particular flavor of Martin-Löf type theory employed in the univalent setting is that this theory has good computational properties. In technical terms, type checking in this 
theory is decidable. Consequently, it is possible to implement the theory on a computer. This is essentially what has been done in the case of the "proof assistants" Coq and Agda. Therefore, mathematics in the univalent setting can be formalized in these systems and the veracity of proofs can be automatically checked. In the case of reasoning involving homotopy theoretic or higher-dimensional algebraic structures, which sometimes involve keeping track of large quantities of complex combinatorial data (think of, e.g., reasoning involving tricategories [25]), being able to make use of the computer to ensure that calculation errors have not been made is potentially quite useful.

Part of the reason that Martin-Löf type theory enjoys such good computational properties is that it is a constructive theory. Classical logic is the usual logic (or framework for organizing mathematical arguments) employed in mathematics (it is the logic of the boolean algebra $\{0,1\}$ ). The logic employed in constructive mathematics is obtained from classical logic by omitting the law of excluded middle, which stipulates that, for any statement $\varphi$, either $\varphi$ or not $\varphi$. Working constructively is often more challenging than working classically and sometimes leads to new developments. Although there are many reasons that one might be interested in pursuing constructive mathematics, we will give several practical reasons. First, constructive mathematics is more general than classical mathematics in the same way that non-commutative algebra is more general than commutative algebra. Second, even in the setting of classical mathematics constructive reasoning can be useful. For example, it is possible to reason constructively in Grothendieck toposes (which do not in general admit classical reasoning). Finally, proofs given in many constructive settings will carry algorithmic content, whereas this is not true in general for proofs given in the classical setting 4

2.8. Reasoning about spaces in type theory. Voevodsky [78] has described a construction of set quotients of types. Explicitly, a relation on a type $X$ is given by a map $R: X \times X \rightarrow$ hProp. For equivalence relations $R$, we can form the quotient $X / R$ of $X$. This type $X / R$ is necessarily a set and can be shown to have the appropriate universal property expected of such a quotient. The set $\pi_{0}(X)$ of path components of $X$ is constructed as a set quotient in the usual way. Because loop spaces $\Omega(X, x)$ of types $X$ at points $x: X$ can be defined type theoretically, it then follows that we may construct all of the higher-homotopy groups $\pi_{n}(X, x)$ of $X$ with basepoint $x: X$ by setting

$$
\pi_{n}(X, x):=\pi_{0}\left(\Omega^{n}(X, x)\right) .
$$

Many of the usual properties of the groups $\pi_{n}(X, x)$ can then be verified type theoretically. E.g.,

(1) The homotopy groups of contractible spaces are 0.

(2) The usual Eckmann-Hilton [22] argument shows that $\pi_{n}(X, x)$ is abelian for $n>1$ (Licata 41] has given a proof of this in the proof assistant Agda).

(3) Voevodsky has developed a large part of the theory of homotopy fiber sequences. Using this it is possible to construct the long exact sequence associated to a fibration.

\footnotetext{
${ }^{4}$ It should be mentioned that there are logical techniques, which themselves exploit the algorithmic content of constructive reasoning, for extracting algorithmic content from classical proofs; see 37.
} 
The notion of inductive type described in Section 2.3 is the type theoretic analogue of the notion of a free algebraic structure on a signature (a list of generating operations together with their arities) as studied in universal algebra. By considering a type theoretic analogue of free algebraic structures on a signature subject to relations, it is possible to describe many familiar spaces type theoretically. This notion is that of higher-inductive type which is currently being developed by a number of researchers (cf., the work by Lumsdaine and Shulman [45, 66] and the recent book [32]). Rather than giving a comprehensive introduction to this subject, we will give a simple example which should illustrate the ideas, and we will then summarize a few of the additional things which can be done with this idea.

To describe the circle $S^{1}$ as a higher-inductive type, we require that it should have one generator $b: S^{1}$ and one generator $\ell: \operatorname{Id}_{S^{1}}(b, b)$. One then obtains an induction principle for $S^{1}$ similar to the induction principle for nat described earlier. Namely, given any type $x: S^{1} \vdash E(x)$ fibered over $S^{1}$ together with terms

- $b^{\prime}: E(b)$ and

- $\ell^{\prime}: \operatorname{Id}_{E(b)}\left(\ell_{*}\left(b^{\prime}\right), b^{\prime}\right)$,

there exists a term $x: S^{1} \vdash \operatorname{rec}_{S^{1}}\left(b^{\prime}, \ell^{\prime}, x\right): E(x)$ (satisfying appropriate "computation" conditions). (Here $\ell_{*}\left(b^{\prime}\right)$ is $b^{\prime}$ transported in the fiber along the loop $l$ for which we refer the reader to Section 6]) So, in particular, in order to construct a map $S^{1} \rightarrow X$, it suffices to give a point $x: X$ and a loop $\ell^{\prime}: \operatorname{Id}_{X}(x, x)$ on $x$. The usual properties of $S^{1}$ then follow from this type theoretic description (see 65 for a type theoretic proof that $\left.\pi_{1}\left(S^{1}, b\right) \cong \mathbb{Z}\right)$.

In basically the same way, finite (and suitably inductively generated) CWcomplexes and relative CW-complexes can be constructed as higher-inductive types. In fact, Lumsdaine 46 has shown that with higher-inductive types, the syntax of type theory gives rise to all of the structure of a model category except for the finite limits and colimits.

\section{Basic Coq constructions}

We will now introduce some basic constructions in Coq and their corresponding homotopy theoretic interpretations. We mention here that there is an accompanying Coq file which includes all of the Coq code discussed here, as well as some additional code 5

3.1. The Coq proof assistant. The Coq proof assistant 9, 13,74 is a computer system which is based on one flavor of Martin-Löf type theory called the calculus of inductive constructions and based in part on the earlier calculus of constructions [19] due to Coquand and Huet.

In February 2010 Voevodsky [78 began writing a Coq library of formalized mathematics based on the univalent model. Pointers to Voevodsky's library and the libraries of other researchers can be found on the Univalent Foundations Wiki [1.

While we will not explain here how to install or process a Coq file, it is nonetheless worth mentioning that the way a Coq file is generally processed is in an active manner. That is, one processes the file in a step-by-step way and as one does so Coq provides feedback regarding the current state of the file.

\footnotetext{
${ }^{5}$ The Coq file can be found either as supplementary data attached to the arXiv version of this paper or on the second author's webpage.
} 

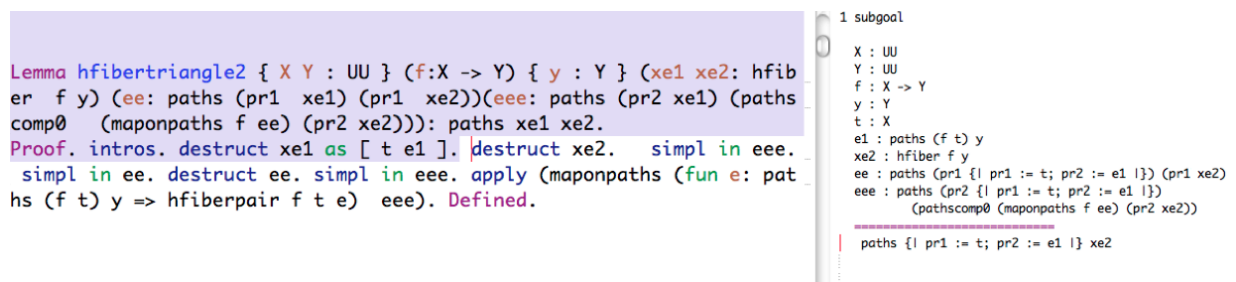

Figure 2. A Coq file being processed.

For example, Figure 2illustrates a Coq file being processed. The user is currently processing a proof (indicated in the left-hand pane of the image) and the shaded text denotes the part of the file which has so far been processed by Coq. The righthand pane is one of two mechanisms which Coq has for providing the user with feedback. In particular, this pane indicates the current state of the proof which is being carried out. Thus, as the user progresses through a proof, the output changes so as to always indicate what remains to be done in order to complete the proof. Further, more detailed, examples of this process are given below.

3.2. Types and terms in Coq. The Coq proof assistant, being based as it is on a form of type theory, allows us to formalize and verify reasoning about types and terms. Throughout our discussion, the reader should have in mind the interpretation of type theory described in Section 2.1. Coq comes with a number of types and type-forming operations already built in. Using these, it is possible to define new types. The first thing we want to do is to select a fixed universe of small types with which we will work. This is accomplished by the following code:

Definition UU := Type.

Here the expression Type is a built-in type in the Coq system which is a universe of types (in a suitable technical sense). The definition above then serves to define UU to be this fixed built-in universe of types. The expression UU is chosen to suggest something like a blackboard bold $U$. We think of the terms of type UU as the small spaces and the universe UU itself as the (large) space of small spaces. Mathematically this is roughly the same as fixing a Grothendieck universe and letting UU be the corresponding space of spaces in the universe.

The type UU corresponds to $\mathcal{U}$ from Section 2.5 above. Henceforth, any statement of the form A : UU should be thought of as asserting that $A$ is a small space.

One interesting feature of the Coq system is that types are themselves terms. In particular, the "type" UU above is itself a term of type Type, where this latter Type is given the index $(n+1)$ when UU has index $n$. That is, being a type is really the same as being a term in a higher universe.

3.3. A direct definition involving function spaces. In order to illustrate some features of the Coq system, we will define some basic constructions on function spaces. First, we define, for any small type $A$, the identity function $1_{A}: A \rightarrow A$ on $A$ :

\section{Definition idfun ( $A$ : UU ) : $A \rightarrow A:=$ fun $x \Rightarrow x$.}

Let us dissect this line of code and try to understand each of its ingredients. A definition, such as this one, is what we will call a direct definition, and such a 
TABle 3. Direct definitions in Coq.



definition has the abstract form summarized (together with the two examples we have so far encountered) in Table 3 .

Several remarks about Table 3 are in order. First, the name is the name given to the term. This can be whatever (modulo some restrictions on the syntactic form) the user likes. The type is the type of the term being defined. I.e., we have that name is of type type. The next thing to note is that the parameters can be a list of variables of fixed types. In the case of idfun there is just a single parameter: the type A : UU; in the case of UU there are no parameters at all. Within a definition, the parameters should be enclosed in brackets as in ( A : UU ). Next, note that it is not strictly necessary to declare the type. When no type is given, Coq will infer the type. Finally, note that the period at the end of the definition must be included in order for Coq to correctly parse the input.

Coming back to the definition of idfun, we would like to remark that the type $\mathrm{A} \rightarrow \mathrm{A}$ is the way of denoting the function space $A^{A}$ in Coq. That is, for types $A$ and $B$, the type $A \rightarrow B$ is the type of functions from A to B. For us, this type should be thought of more specifically as the type of all continuous functions from the space A to the space B. The remaining part of this definition is the actual content of the definition: fun $\mathrm{x}=>\mathrm{x}$. In this definition, the expressions fun and $\Rightarrow$ go together and tell us that it is the function $\lambda_{x: A} x$ in the notation of Section 2.2. So this function takes a point $x$ in $A$ and returns $x$ itself. I.e., fun . . $\Rightarrow>$. is the same as giving a definition of a function by writing $\ldots \mapsto \ldots$.

3.4. An indirect definition involving function spaces. We will now show, given functions $f: A \rightarrow B$ and $g: B \rightarrow C$, how to construct the composite $g \circ f: A \rightarrow C$ type theoretically. In order to introduce indirect definitions, we will give two ways to construct $g \circ f$.

First, we have the following direct definition:

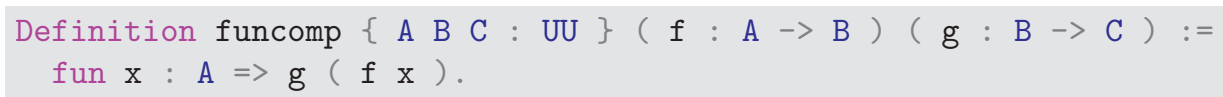

Notice about this definition that we have enclosed the first three parameters in curved brackets as $\{A B C$ : UU\} in order to indicate to the Coq system that these parameters are implicit. Implicit parameters do not need to be supplied (when the term is applied), by the user, and the system will try to infer the values of these parameters. In this case, these can be inferred from the types of $f$ and $g$. Note also that we have here not given explicitly the type of the term being defined. As such, we must include the additional typing data $\mathrm{x}$ : $\mathrm{A}$ in the definition in order for the Coq system to be able to infer the type of the term.

The utility of indirect definitions in Coq is that sometimes it is not easy to see how to give the explicit definition of a term. This is especially true as one starts working with increasingly complicated definitions. As such, rather than having to struggle to define exactly the required term, it is possible to construct the term being defined as a kind of proof. Along the way, as this proof is constructed, certain 
automation possible in Coq can be employed. In order to see how this works in practice, let us introduce our first indirect definition.

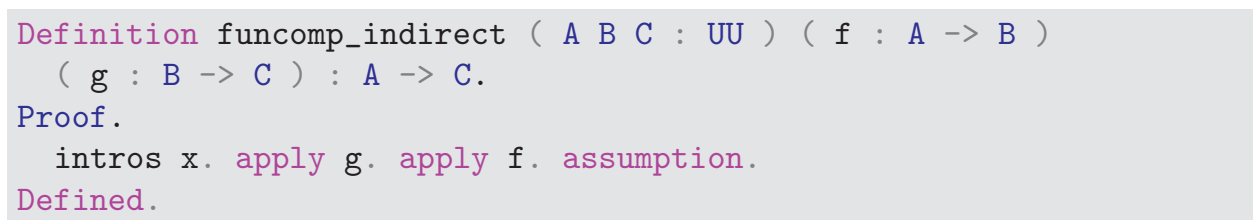

The first observation about this definition is that it looks like everything to the left of the $:=$ in a direct definition. In this case there are three parameters of type UU (namely, A, B and C). There is also one parameter of type A $\rightarrow$ B (namely, $f$ ). Finally, there is one parameter of type B $\rightarrow$ C (namely, g).

After the first full stop of an indirect definition, we encounter the start of the proof. This is given by the line

\section{Proof.}

Likewise, the end of the proof is indicated by

\section{Defined.}

Between the start of the proof and the end of the proof is a sequence of what are called tactics, which allow one to construct, using the given parameters, the required term. One limitation of writing an article which includes proofs in Coq, is that proofs in Coq are usually constructed using "backward" reasoning, and so it can be hard to read for the uninitiated. In particular, the nature of Coq is such that, qua interactive proof assistant, proofs can be understood better by directly watching the output of a Coq session, where an additional window appears after each step, giving us precise explanations of any given step of the proof. We have included in Figure 3 the output from Coq as we move through the proof of funcomp_indirect. Readers should not be discouraged if they are unable to read Coq proofs directly: it is much easier if you are going through the proofs yourself in the computer.

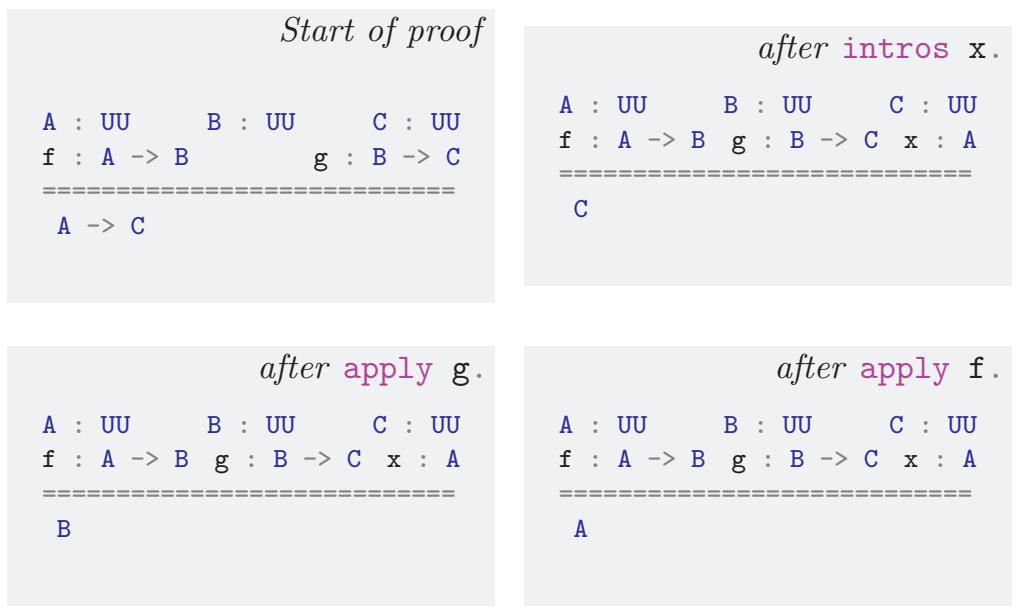

Figure 3. Coq output during indirect definition of function composition. 
We will now go through the proof one step at a time. Notice that once the proof is started, Coq displays the hypotheses (above the line $=======$ ) together with the current goal (below the line). Since the goal is to construct a function A $\rightarrow$ C, we are allowed to assume given an arbitrary term $\mathrm{x}$ : A. This is accomplished in Coq by entering intros $\mathrm{x}$. Note that the name $\mathrm{x}$ here is something which we have chosen and the user can choose this name freely (or it can be omitted, in which case the Coq system will supply a name of its own choosing). As such, after processing intros $x$, the output has changed (as indicated in Figure 3) by adding a new hypothesis ( $\mathrm{x}: \mathrm{A}$ ) and changing the goal to $\mathrm{C}$. This means that we now need to supply a term of type $\mathrm{C}$. To accomplish this, we note that we are given a function $\mathrm{g}: \mathrm{B} \rightarrow \mathrm{C}$, and so it suffices to supply a term of type B. We communicate the fact that we intend to use $g$ to obtain the goal by entering apply $g$. The effect of this is to change the goal from C to B since B is the domain of $\mathrm{g}$. Applying the same reasoning now with $f$, we are in the final situation indicated in Figure 3 . Because we have as a hypothesis the term $\mathrm{x}$ : $\mathrm{A}$ and the current goal is to construct a term of type A, we may simply communicate to the Coq system that there is already a term of the required type appearing among the hypotheses. This is accomplished by entering assumption. Indeed, at this point, Coq tells us

No more subgoals.

and the proof is complete. Note that we must add the final "Defined." in order for the Coq system to correctly record the proof.

We can now check that our definition coincides with the direct one by entering

Print funcomp.

Print funcomp_indirect.

The effect of Print is to output both the type and explicit definition of the term in question. In particular, even if the term in question was defined indirectly, as our funcomp_indirect was, it is an explicit term as far as Coq is concerned, and when Print is used, Coq will unfold the term to give a completely explicit description.

To understand what this means, simply think of a linear differential equation for which it is possible to explicitly write down the solutions. The solution set can be difficult to write down, but it can be done. Although you may not want to have these solutions in front of you, you know that the explicit solutions are available if they are required (say, to verify that they satisfy a certain equation). The Coq system effectively keeps track of this kind of bookkeeping for the user.

\section{Some BASIC InduCtive types}

In this section we will begin manipulating inductive types in Coq. We start with the natural numbers as a warm-up and then move on to consider total spaces of fibrations (dependent sums).

4.1. The inductive type of natural numbers. The inductive type nat of natural numbers is already defined in the Coq system. As an inductive type, nat is generated by

- the single generator (0-ary operation) 0 : nat and

- the single generating function (1-ary operation) $\mathrm{S}$ : nat $\rightarrow$ nat (this is the usual successor function). 
In Coq, this inductive type is specified as follows:

Inductive nat $:=0 \mid S:$ nat $\rightarrow$ nat.

Here the internal Coq command Inductive functions similarly to Definition (as we will see below). For now the crucial point is to observe that the generating operations of the inductive type appear on the right of := and are separated by the symbol $\mid$.

One of the advantages of working with an inductive type such as the natural numbers is that functions with inductive domain can be defined by cases. To see an example, consider the predecessor function:

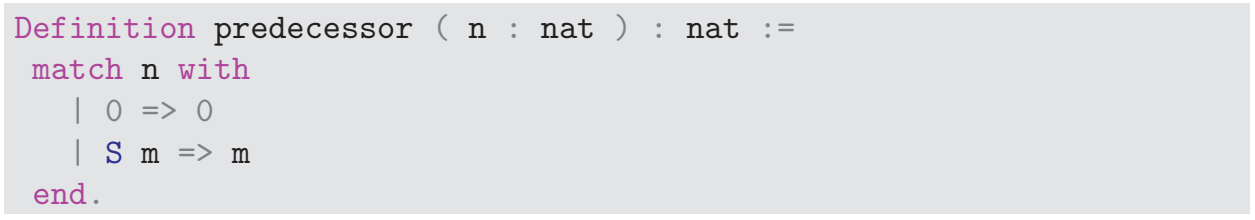

This is the way of telling Coq that the predecessor function is the function nat $\rightarrow$ nat given by case analysis as

$$
\operatorname{predecessor}(n):= \begin{cases}0 & \text { if } n=0, \text { and } \\ m & \text { if } n=(m+1) .\end{cases}
$$

The definition of the predecessor function given above using match is a direct definition as described above in Section 3.3 It is also possible to define the predecessor function via an indirect definition as follows:



In the proof there are two new tactics. The first, destruct n, tells the Coq system that we will reason by cases on the structure of $n$ as a term of type nat. Coq knows that, as a natural number, there are two cases, and in the first case there is no hypothesis necessary (see Figure 4) because $\mathrm{n}$ is 0 in this case. At this stage, we know that we would like the output of the function to be 0 , and we tell Coq this using the exact tactic. In general, if we enter exact $x$, this tells Coq that the term we are looking for is exactly the term $x$. Once we have entered exact 0 , Coq moves on to the second possibility: the term is a successor. Note that in the list of hypotheses at this stage (see Figure 4) the term $n$ : nat is listed and so superficially things are just as they were at the start of the proof. However, because we earlier employed the destruct tactic, Coq knows that we must now give the required output of the predecessor function when given the value $\mathrm{S} n$. As such, we enter exact $\mathrm{n}$. Comparing predecessor and indirect_predecessor using the Print command reveals that they are indeed identical terms. We will now turn to several of the inductive types more closely related to the homotopy theoretic side of things.

4.2. Fibrations and the total space of a fibration. Fibrations can be understood as a homotopy theoretic generalization of the notion of a fiber bundle. Similarly, they can be understood as a homotopy theoretic version of Grothendieck fibrations familiar from category theory and algebraic geometry. In particular, for 


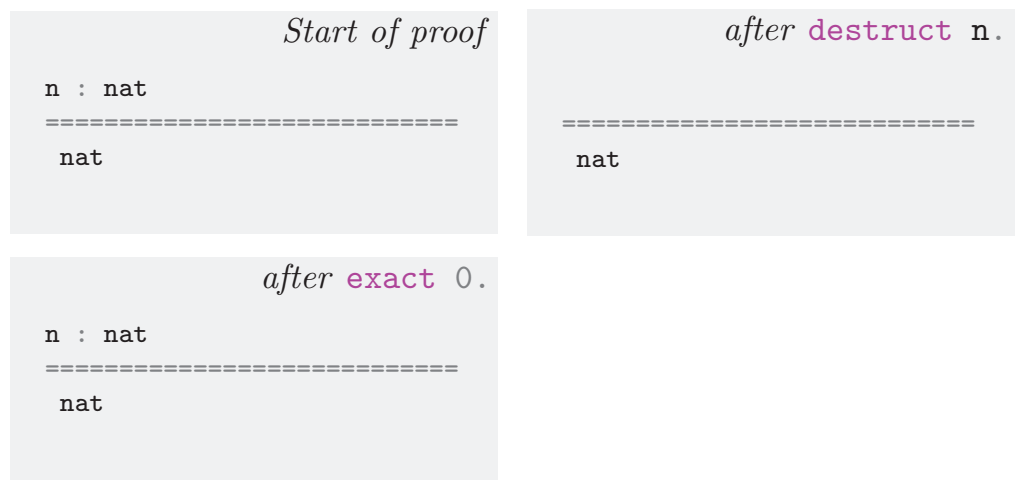

Figure 4. Coq output during indirect definition of the predecessor function.

spaces, a fibration is a map

$$
\pi: E \rightarrow B
$$

which possesses a certain homotopy-lifting property. In this case we would refer to $B$ as the base space and to $E$ as the total space of the fibration. Given a point $b$ in $B$, the fiber over $b$, which we sometimes write as $E_{b}$, is just the preimage $\pi^{-1}(b)$ of $b$ under the map $\pi$. As mentioned in Section 2.1 above, fibrations correspond to types which depend on parameters (so-called dependent types). In Coq, the way of dealing with dependent types is somewhat different from the one sketched in Section 2.1. In particular, given a small type B : UU, a fibration over B is represented by the corresponding term

E : $B \rightarrow$ UU.

The idea that a fibration over a base space $B$ can be represented as a map from $B$ into a suitable universe is a classical idea, especially in category theory where it is a basic fact that Grothendieck fibrations over a category $B$ correspond to pseudofunctors from $B$ into the 2-category of small categories. (These ideas are ubiquitous in category theory, and a nice exposition can be found in the first section of [70].)

The idea behind this correspondence is that a fibration can be completely recovered from its base space $B$ together with its fibers by gluing the fibers together in a coherent way in accordance with the structure of the base space. The same can be accomplished in Coq by defining the total space of a fibration $\mathrm{E}: \mathrm{B} \rightarrow \mathrm{UU}$ as an inductive type.

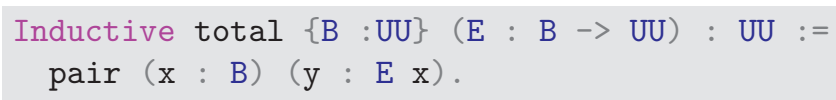

Intuitively, total $E$ should be thought of as a space consisting of all pairs ( $b, e)$, where $\mathrm{b}$ is a point of the base space $\mathrm{B}$ and $\mathrm{e}$ is a point of the fiber $\mathrm{E} \mathrm{b}$ over $\mathrm{b}$. In the literature on type theory such total spaces are known as dependent sums and are often denoted using summation notation as $\sum_{x: B} E(x)$. From the point of view of logic, the total space corresponds to existential quantification.

Fiber bundles $E \rightarrow B$ are sometimes thought of as a "twisted" generalization of direct products $F \times B \rightarrow B$, and the fact that fibrations are a homotopical generalization of this notion reveals itself type theoretically by the fact that the 
total space construction total is a generalization of the construction of direct products of spaces $A \times B$, which are given by

\section{Definition dirprod $\{A B$ : $U U$ : $U U$ : $=$ total (fun $x: A \Rightarrow B$ ).}

Returning to total, we define a projection map total $\mathrm{E} \rightarrow \mathrm{B}$ by

Definition $\operatorname{pr} 1\{\mathrm{~B}: \mathrm{UU}\}\{\mathrm{E}: \mathrm{B} \rightarrow \mathrm{UU}\}:$ total $\mathrm{E} \rightarrow \mathrm{B}:=$

fun $\mathrm{z} \Rightarrow$ match $\mathrm{z}$ with pair $\mathrm{x} \mathrm{y} \Rightarrow \mathrm{x}$ end.

This map serves to exhibit $\mathrm{E}$ as a fibration over B. In type theoretic notation, pr1 is the projection $\pi_{1}: \sum_{x: B} E(x) \rightarrow B$.

\section{THE PATH SPACE}

As mentioned in Sections 2.3 and 2.4, the type theoretic identity type is interpreted homotopy theoretically as the path space. The path space, and the corresponding type in Coq, is so important that we will now carefully describe several basic constructions involving it in the setting of Coq.

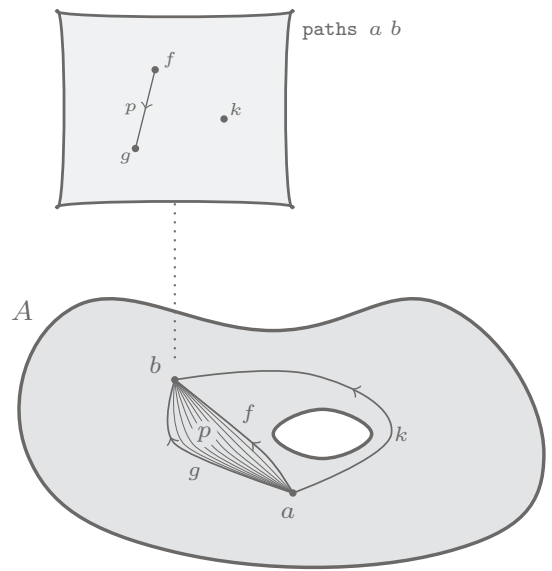

FiguRE 5. The path space fibration paths $a$ with the fiber over a point $b$. In the base $p$ is a (path) homotopy from $f$ to $g$ and in the fiber it is a path from $f$ to $g$.

In Coq, the path space paths is defined as

\section{Notation paths := identity}

Here identity, like nat, is a built-in inductive type in the Coq system. We can see how it is defined inductively using Print to find

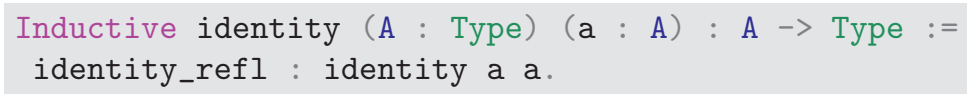

That is, for each a : A, identity a is the fibration freely generated by a term identity_refl a in the fiber over a.

We add the following line in order to introduce a slightly shorter notation for the terms identity_refl:

Notation idpath := identity_refl. 
That is, for a : A, idpath a : paths a a is the identity path based at a.

Recall that a path in a space $A$ is a continuous function $\varphi: I \rightarrow A$ where $I=[0,1]$ is the unit interval. We say that $\varphi$ is a path from a point a of $A$ to a point $b$ of $A$ when $\varphi(0)=a$ and $\varphi(1)=b$. Then the path space $A^{I}$ is the space of paths in $A$, and it comes equipped with two maps $\partial_{0}, \partial_{1}: A^{I} \rightarrow A$ given by $\partial_{i}(\varphi):=\varphi(i)$ for $i=0,1$. The induced map $\left\langle\partial_{0}, \partial_{1}\right\rangle: A^{I} \rightarrow A \times A$ is a fibration which gives a factorization of the diagonal $\Delta: A \rightarrow A \times A$ as

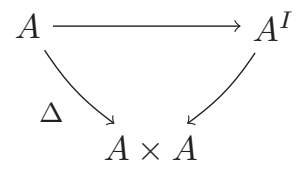

where the first map $A \rightarrow A^{I}$ is a weak equivalence in the sense of homotopy theory and the second is the fibration mentioned above. Here the first map $A \rightarrow A^{I}$ sends a point $a$ to the constant loop based at $a$. (That is, this first map is precisely idpath.) One of the many important contributions of Quillen in 60. was to demonstrate that it is in fact possible to do homotopy theory without the unit interval provided that one has the structure of path spaces, weak equivalences, fibrations, and a few other ingredients. This is part of the reason that, even though type theory does not (without adding higher-inductive types or something similar) provide us with a unit interval, it is still possible to work with homotopy theoretic structures type theoretically.

5.1. Groupoid structure of the path space. We will now describe the groupoid structure which the path space can endow on types. The connection between these constructions and groupoids were first observed by Hofmann and Streicher [29].

First, given a path $f$ from $a$ to $b$ in $A$ we would like to be able to reverse this path to obtain a path from $b$ to $a$. For topological spaces this is easy because a path $\varphi: I \rightarrow A$ gives rise to an inverse path $\varphi^{\prime}$ given by $\varphi^{\prime}(t):=\varphi(1-t)$, for $0 \leq t \leq 1$.

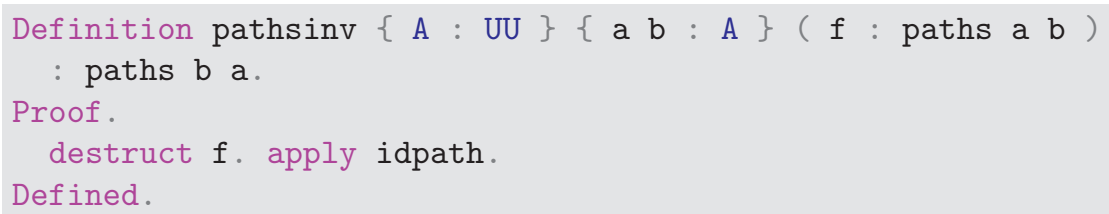

Here recall that destruct allows us to argue by cases about terms of inductive types. Here $f$ is of type paths $a b$, which is inductive, and therefore this tactic applies. In this case, there is only one case to consider: $f$ is really the identity path idpath a : paths a a. Because the inverse of the identity is the identity, we then use apply idpath to complete the proof. (Note that we could also have used exact ( idpath a ) instead of apply idpath here to obtain the same term.)

Next, given a path $f$ as above together with another path $g$ from $b$ to $c$, we would like to define the composite path from $a$ to $c$ obtained by first traveling along $f$ and then traveling along $g$. This operation of path composition is defined as follows:

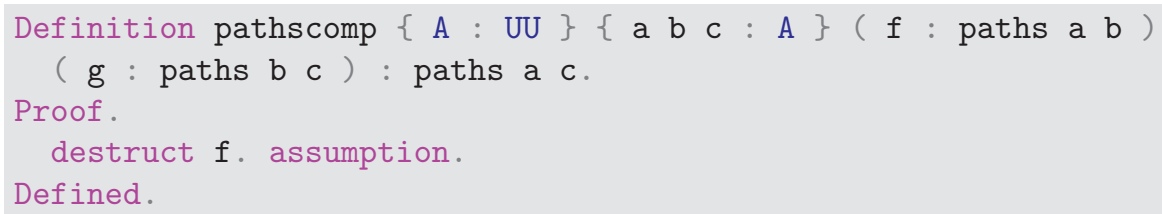


Once again, the proof begins with destruct $f$ which effectively collapses $f$ to a constant loop. In particular, the result of this is to change the ambient hypotheses so that $\mathrm{g}$ is now of type paths a $\mathrm{c}$ (see Figure 6). At this stage, the goal matches the type of $\mathrm{g}$, and we use assumption to let the Coq system choose $\mathrm{g}$ as the result of composing g with the identity path.

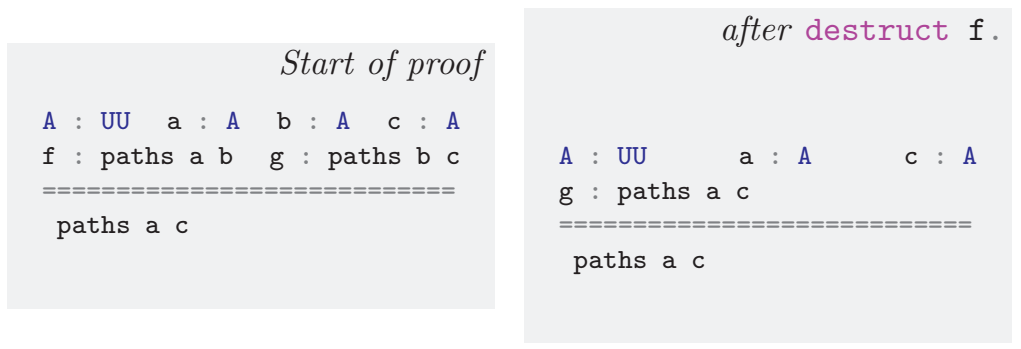

Figure 6. Coq output during the definition of path composition.

One immediate consequence of this definition is that the unit law $f \circ 1_{a}=f$ for $f: a \rightarrow b$ holds on the nose in the sense that the terms pathscomp (idpath a) $f$ and $\mathrm{f}$ are identical in the strong $=$ sense. On the other hand, the unit law $1_{b} \circ f=f$ does not hold on the nose. Instead, it only holds up to the existence of a higherdimensional path as described in the following lemma:

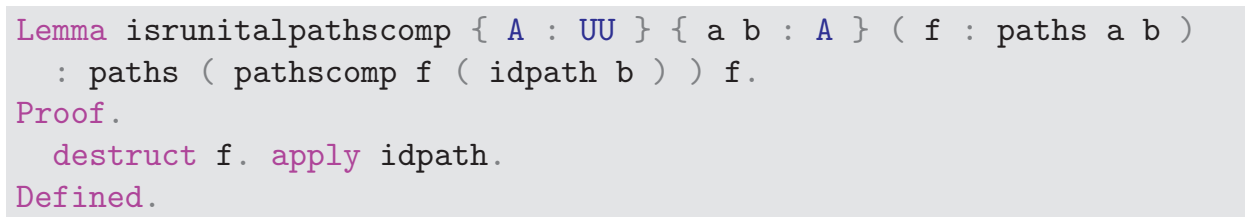

The proof of this requires little comment (when $f$ becomes itself an identity path, the composite becomes, by the left-unit law mentioned above, an identity path). The one thing to note is that here instead of Definition we have written Lemma. Although there are some technical differences between these two ways of defining terms, they are for us entirely interchangeable, and therefore we use the appellation "Lemma" in keeping with the traditional mathematical distinction between definitions and lemmas.

That fact is, up to the existence of higher-dimensional paths, composition of paths is associative and the inverses given by pathsinv are inverses for composition are recorded as the terms isassocpathscomp, islinvpathsinv, and isrinvpathsinv. However, the descriptions of these terms are omitted in light of the fact that they all follow the same pattern as the proof of isrunitalpathscomp.

5.2. The functorial action of a continuous map on a path. Classically, given a continuous map $f: A \rightarrow B$ and a path $\varphi: I \rightarrow A$ in $A$, we obtain a corresponding path in $B$ by composition of continuous functions. Thinking of spaces as $\infty$-groupoids, this operation of going from the path $\varphi$ in $A$ to the path $f \circ \varphi$ in $B$ is the functorial action of $f$ on 1 -cells of the $\infty$-groupoid $A$. In Coq, this action of transporting a path in $A$ to a path in $B$ along a continuous map is given as follows: 


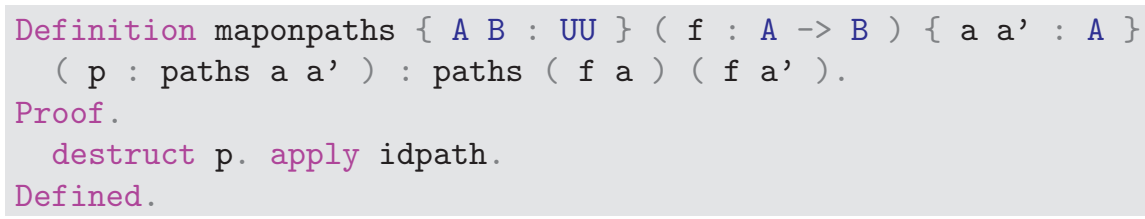

The proof again follows the familiar pattern: when the path $p$ is the identity path on $a$, the result of applying $f$ should be the identity path on $f(a)$. We introduce the following notation for maponpaths:

\section{Notation " $f$ ' $p$ " := ( maponpaths $f \mathrm{p}$ ) (at level 30 ).}

This is an example of a general mechanism in Coq for defining notations, but discussion of this mechanism is outside of the scope of this article (the crucial point here is that the value 30 tells how tightly the operation should bind).



FIGURE 7. Representation of maponpaths.

We leave it as an exercise for the reader to verify that the operation maponpaths respects identity paths, as well as composition and inverses of paths.

\section{TRANSPORT}

Given a fibration $\pi: E \rightarrow B$ together with a path $f$ from $b$ to $b^{\prime}$ in the base $B$, there is a continuous function $f_{*}: E_{b} \rightarrow E_{b^{\prime}}$ from the fiber $E_{b}$ of $\pi$ over $b$ to the fiber $E_{b^{\prime}}$ over $b^{\prime}$. This operation $f_{*}$ of forward transport along a path (see Figure 8) is described in Coq as follows:

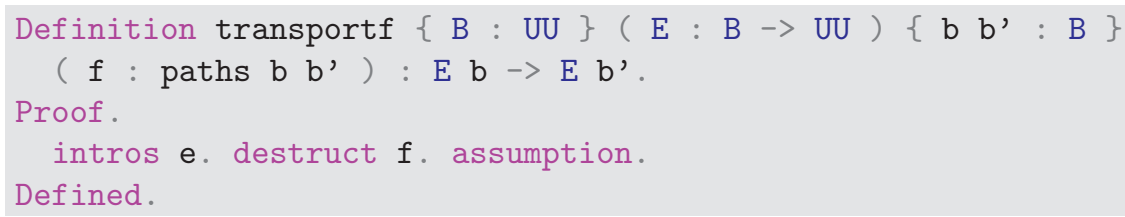




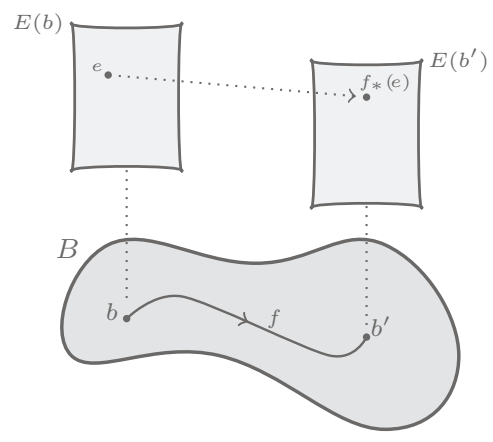

FiguRE 8. Forward transport.

For a path $f$ as above, there is a corresponding operation $f^{*}: E_{b^{\prime}} \rightarrow E_{b}$ of backward transport, and it turns out that $f_{*}$ and $f^{*}$ constitute a homotopy equivalence.

We will now briefly discuss homotopy and homotopy equivalence in the setting of Coq before returning to forward and backward transport in Section 6.2.

6.1. Homotopy and homotopy equivalence. Recall that for continuous functions $f, g: A \rightarrow B$, a homotopy from $f$ to $g$ is given by a continuous map $h: A \rightarrow B^{I}$ such that

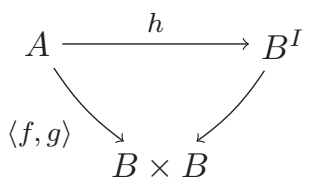

commutes.

In Coq, the type of homotopies between functions $f, g: A \rightarrow B$ is given by

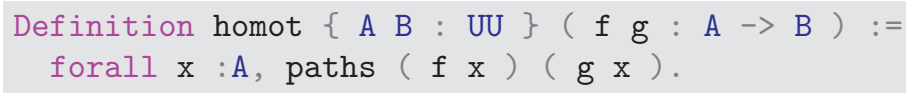

Here we encounter a new ingredient in Coq: the dependent product forall. I.e., forall $\mathrm{x}: \mathrm{B}, \mathrm{E} \mathrm{x}$ is Coq's way of writing the dependent product $\prod_{x: B} E(x)$ described in Section 2.2. One particular consequence of this is that if we are given a term

$\mathrm{s}:$ ( forall $\mathrm{x}: \mathrm{B}, \mathrm{Ex}$ )

and another term $\mathrm{b}: \mathrm{B}$, then the term $\mathrm{s}$ can be applied to the term $\mathrm{b}: \mathrm{B}$ to obtain a term of type $\mathrm{E} b$. The result of applying $\mathrm{s}$ to $\mathrm{b}$ is denoted by

$\mathrm{s} b: \mathrm{E} b$.

I.e., $\mathrm{s} \mathrm{b}: \mathrm{E} \mathrm{b}$ is Coq's way of writing the application $\operatorname{app}(s, b): E(b)$ from Section 2.2. Below we have more to say about forall.

Now, a map $f: A \rightarrow B$ is a homotopy equivalence when there exists a map $f^{\prime}: B \rightarrow A$ together with homotopies from $f^{\prime} \circ f$ to $1_{A}$ and from $f \circ f^{\prime}$ to $1_{B}$. In this case, we say that $f^{\prime}$ is a homotopy inverse of $f$. Two spaces $A$ and $B$ are said to have the same homotopy type when there exists a homotopy equivalence $f: A \rightarrow B$. 
In Coq, we define the type of proofs that a map $f: A \rightarrow B$ is a homotopy equivalence as follows:

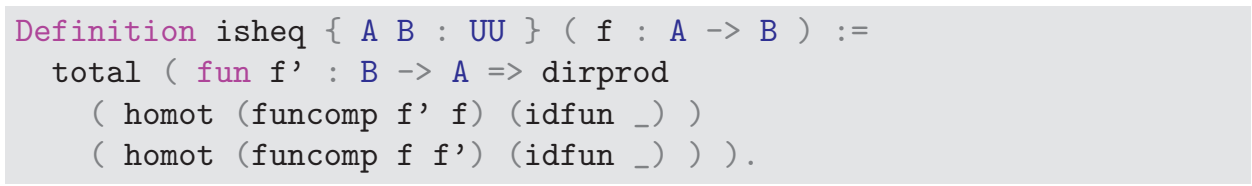

Let us pause for a moment to consider the meaning of the type isheq. Intuitively, isheq $f$ is the type consisting of the data which one must provide in order to prove that $f$ is a homotopy equivalence. That is, a term of type isheq $f$ consists of

- a continuous map f' : $B \rightarrow$ A,

- a homotopy from funcomp $f$ ' $f$ to the identity on $B$,

- a homotopy from funcomp $f f^{\prime}$ to the identity on $A$.

Indeed, by the definitions of total and dirprod the terms of isheq $f$ can be regarded as a tuple of such data. In type theoretic notation, isheq $f$ would therefore be denoted by $\sum_{f^{\prime}: B \rightarrow A}\left(\operatorname{homot}\left(f^{\prime} \circ f\right) 1_{A}\right) \times\left(\operatorname{homot}\left(f \circ f^{\prime}\right) 1_{B}\right)$.

6.2. Forward and backward transport. It turns out that, as mentioned above, the backward transport map $f^{*}: E_{b^{\prime}} \rightarrow E_{b}$ is a homotopy inverse of forward transport $f_{*}$. Denote by transportb the backward transport term. It is often convenient to break up the proofs of larger facts into smaller lemmas, and we will do just this in order to show that transportf $\mathrm{E} f$ is a homotopy equivalence. In particular, we begin by proving that $f_{*} \circ f^{*}$ is homotopic to the identity $1_{E_{b^{\prime}}}$ :



Next, we prove that $f^{*} \circ f_{*}$ is homotopic to the identity $1_{E_{b}}$ as forthandback (we omit the proof because it is identical to the proof of backandforth):

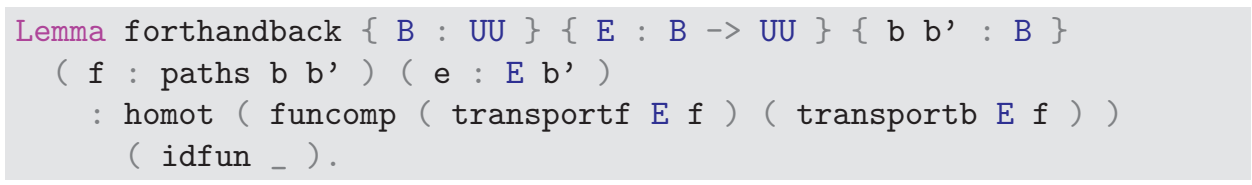

Using these lemmas we can finally prove that transportf $\mathrm{E} f$ is a homotopy equivalence.

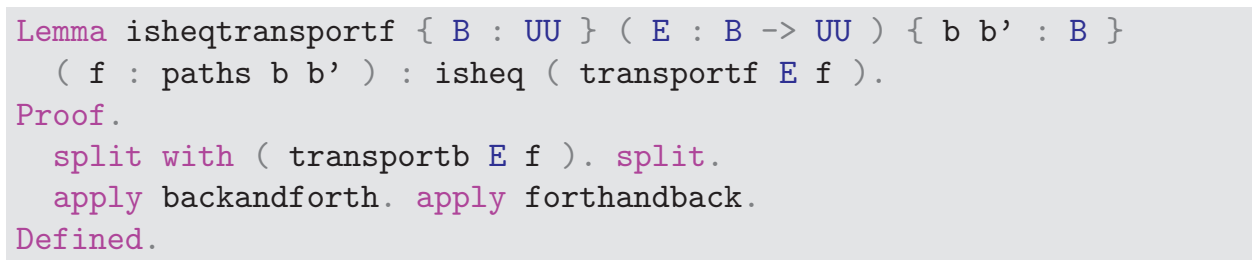




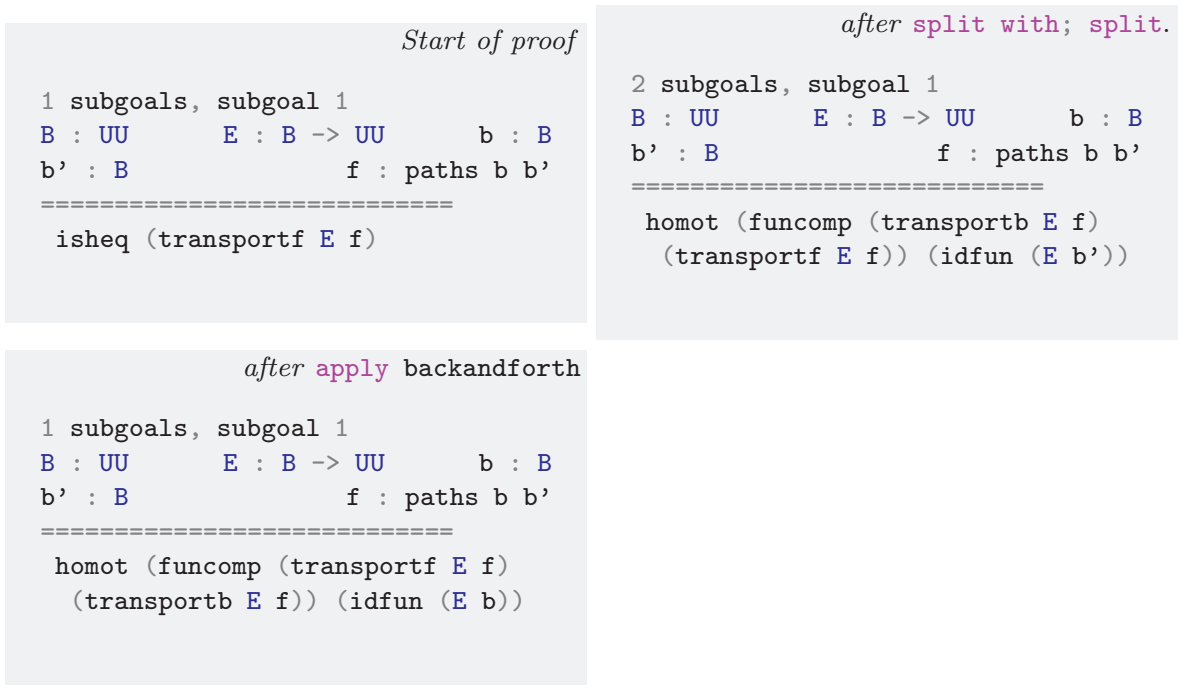

Figure 9. Coq output during the proof that forward transport is a homotopy equivalence.

There are several points to make about this proof. The initial goal is to supply a term of type isheq ( transportf $\mathrm{E} f$ ). Now, this type is itself really of the form

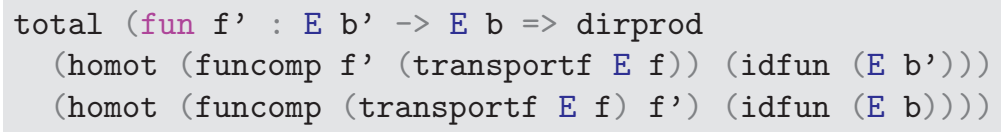

(you can see this in the proof by entering unfold isheq), and in general to construct a term of type total E, for $\mathrm{E}: \mathrm{B} \rightarrow \mathrm{UU}$, it suffices (by virtue of the definition of total) to give a term $\mathrm{b}$ of type $\mathrm{B}$ together with a term of type $\mathrm{E} \mathrm{b}$. This is captured in Coq by the command split with, and one should think of split with $\mathrm{b}$ as saying to Coq that you will construct the required term using $\mathrm{b}$ as the term of type B you are after. Upon using this command, the goal will automatically be updated to $E$ b. In this case, entering split with (transportb $E \mathrm{f}$ ) is the way to tell Coq that we take transportb $\mathrm{E} f$ to be the homotopy inverse of transportf $\mathrm{E} f$. So, after entering this command the new goal becomes

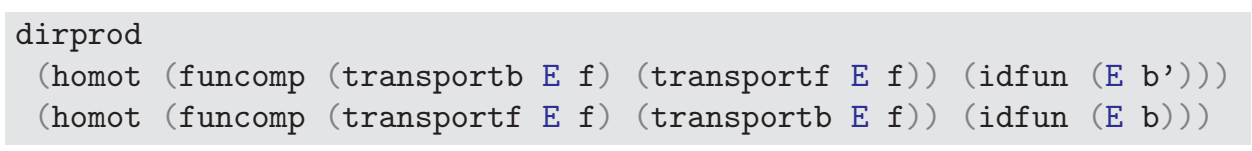

As with total $\mathrm{E}$, in order to construct a term of type dirprod A B, it suffices to supply terms of both types A and B. When given a goal of the form dirprod A B, we use the split tactic to tell Coq that we will supply separately the terms of type A and B individually (as opposed to providing a term by some other means). (See Figure 9 for the result of applying both split with and split in the particular proof we are considering.)

The final new ingredient from the proof of isheqtransportf is the appearance of the tactic apply. When you have proved a result in Coq and you are later given a goal which is a (more or less direct) consequence of that the result, then the tactic 
apply will allow you to apply the result. In this case, the lemmas backandforth and forthandback are exactly the lemmas required in order to prove the remaining subgoals.

6.3. Paths in the total space. Using transport it is possible to give a complete characterization of paths in the total space of a fibration E : B $\rightarrow$ UU. Along these lines, the following lemma gives sufficient conditions for the existence of a path in the total space:



This lemma shows that, given points $\mathrm{x}$ and $\mathrm{y}$ of the total space, in order to construct a path from $\mathrm{x}$ to $\mathrm{y}$ it suffices to provide the following data:

- a path $\mathrm{f}$ from pr1 $\mathrm{x}$ to $\operatorname{pr} 1 \mathrm{y}$; and

- a path $g$ from the result of transporting pr2 $\mathrm{x}$ along $\mathrm{f}$ to pr2 $\mathrm{y}$.

This is illustrated in Figure 10 in the special case where $\mathrm{x}$ is the pair pair $\mathrm{b}$ e and $y$ is the pair pair b' $e^{\prime}$.

Regarding the proof of pathintotalfiber, note that the effect of applying destruct $\mathrm{x}$ as $[\mathrm{x} 0 \mathrm{x} 1]$ is that it tells Coq that we would like to consider the case where $\mathrm{x}$ is really of the form pair $\mathrm{x} 0 \mathrm{x} 1$. The only new tactic here is simpl in $*$ which tells Coq to make any possible simplifications to the terms appearing in the goal or hypotheses. For example, in this case, Coq will simplify (pr1 (pair $\mathrm{x} 0 \mathrm{x} 1)$ ) to $\mathrm{x} 0$.

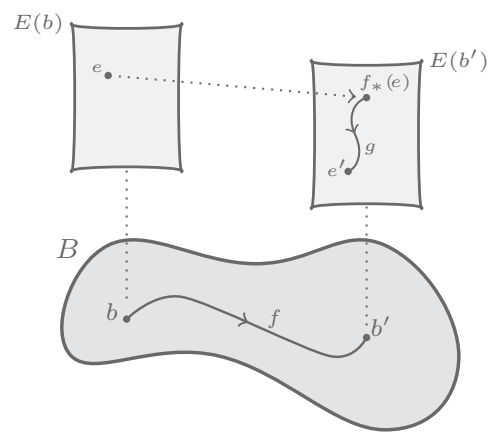

Figure 10. Paths in the total space.

On the other hand, if we are given a path $\mathrm{f}$ from $\mathrm{x}$ to $\mathrm{y}$ in the total space, there is an induced path in the base given by

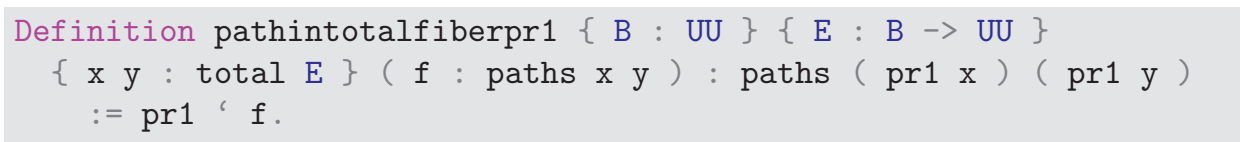


Furthermore, we may transport pr2 $\mathrm{x}$ along pathintotalfiberpr $1 \mathrm{f}$, and there is a path from the resulting term to pr2 y:

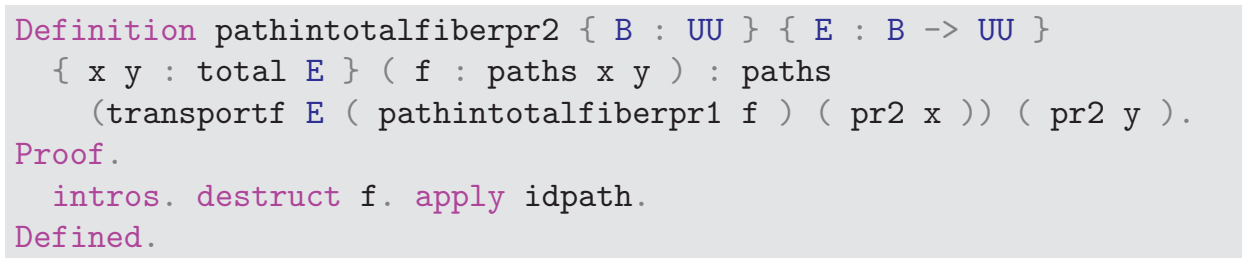

Finally, we prove that every path in the total space is homotopic to one obtained using pathintotalfiber:

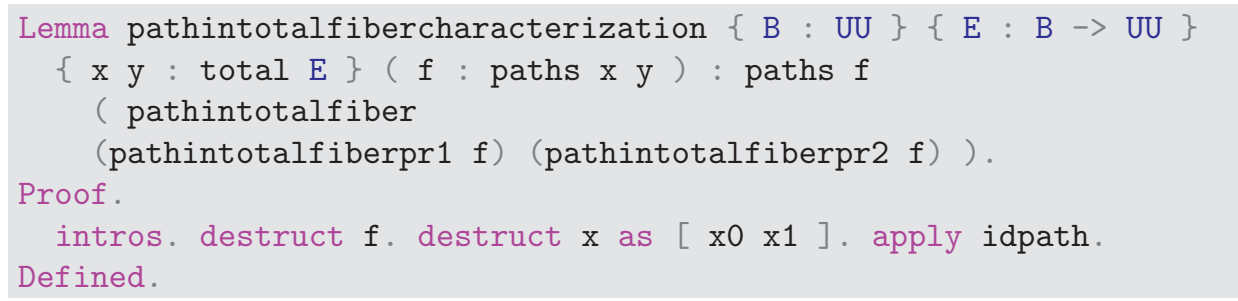

\section{WEAK EQUIVALENCES AND HOMOTOPY EQUIVALENCES}

In this section we introduce further basic homotopy theoretic notions including the crucial notion of weak equivalence. Classically, weak equivalences are those maps which induce isomorphisms on (all higher) homotopy groups. Here we work with an alternative definition of weak equivalence given by Voevodsky which has the advantage of not requiring homotopy groups. Between sufficiently nice spaces, weak equivalences turn out to coincide with homotopy equivalences, and the main goal of this section is to prove this fact in Coq. The Coq proofs in this section become increasingly sophisticated, and accordingly we will begin to pass quickly over the more basic features of the proofs while at the same time giving their mathematical interpretations.

7.1. Contractibility. A space $A$ is said to be contractible when the canonical map $A \rightarrow 1$ to the one point space is a homotopy equivalence. In Coq, the notion of contractibility is captured by the following definition:

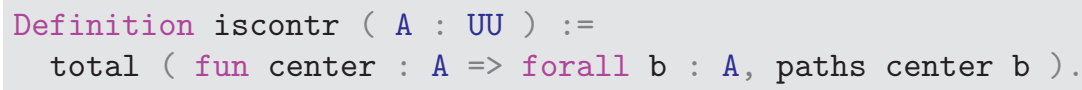

That is, a term of type iscontr A consists of the data required to prove that A is contractible:

- a point center of $\mathrm{A}$, which we will sometimes refer to as the center of contraction; and

- a continuous assignment of, to each b : A, a path from center to b.

There are various facts about contractible spaces (e.g., 1 is contractible, contractible spaces satisfy the principle of "proof irrelevance", and so forth) which are ready consequences of this definition. However, we will leave the investigation of such matters to the reader and merely include one important fact about contractible spaces: homotopy retracts of contractible spaces are contractible. That is, given continuous functions $r: A \rightarrow B$ and $s: B \rightarrow A$ together with a homotopy $p$ as 
indicated in (7.1)

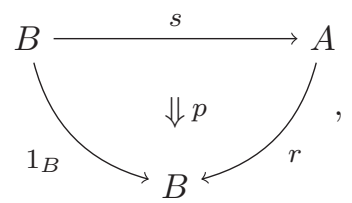

if the space $A$ is contractible, then so is the space $B$. Note that in (7.1) we employ the convention, familiar from higher-dimensional category theory, of indicating the homotopy $p$ by a double arrow $\Rightarrow$. In Coq:

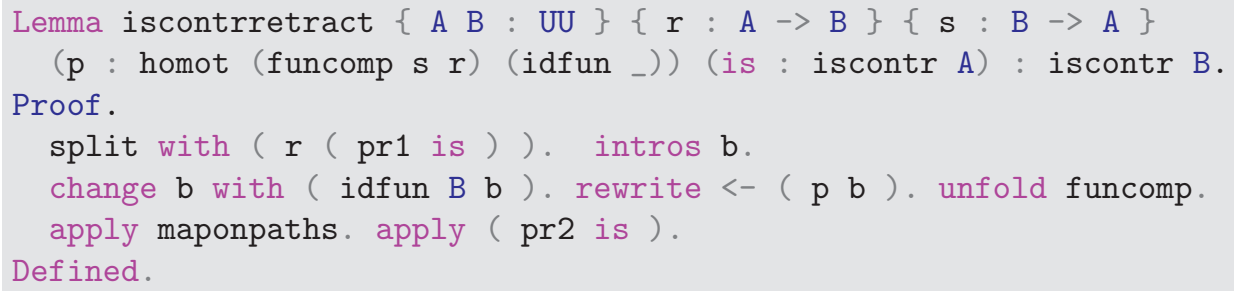

The output of Coq during the proof can be found in Figure 11. First, we need to provide a center of contraction for B. The center of contraction for A is the term pr1 is. By entering split with ( $r(p r 1$ is ) ) we tell Coq to take the center of contraction of B to be the term $r$ ( $p r 1$ is ). After this, the goal becomes

forall b: B, paths $(r(\operatorname{pr} 1$ is $)) b$

which is to say that we must prove that there is a path from $r$ ( $\operatorname{pr} 1$ is) to any other point of B. Next, after using intros b, we enter the command

change $b$ with ( idfun $B$ b )

to replace the term $\mathrm{b}$ in the goal with the equal term idfun $\mathrm{B}$ b. Observe that the term $\mathrm{p}$ b has type

paths (funcomp s $r$ b) (idfun $B$ b).

The tactic rewrite allows us to replace each occurrence of a term in the goal by a term which is in the same path component. In this case, because $\mathrm{p} b$ is a path from funcomp $s \mathrm{r} b$ to idfun $B \mathrm{~b}$, the result of rewriting with rewrite $<-(\mathrm{p} b)$ is to replace each occurrence of idfun $B$ b in the goal by funcomp $s \quad r$ b. Here the backward arrow $<-$ indicates that we are rewriting the path $\mathrm{p} b$ from right to left. 
The homotopy fiber of $f$ over $b$ is the homotopical analogue of the ordinary fiber $f^{-1}(b)$. So, a typical point of the homotopy fiber is a pair consisting of a point $a$ of $A$ together with a path

$$
f(a) \longrightarrow-b
$$

in $B$.

There are various reasons for considering homotopy fibers. Homotopy fibers play a role for fibrations analogous to that played by ordinary fibers for fiber bundles. For us, the interest in homotopy fibers comes from their presence in the definition of weak equivalences given below.

From the point of view of category theory, the homotopy fiber of $f$ over $b$ is the $\infty$-groupoid version of the comma category $(f \downarrow b)$. As in the categorical setting, there are actions of the operation of taking the homotopy fiber over a fixed point on maps over the base $B$. That is, given continuous maps $f: A \rightarrow B, g: C \rightarrow B$ and $h: A \rightarrow C$ together with a homotopy $p$ from $g \circ h$ to $f$, there is an induced map

$$
\text { hfiber } f b \rightarrow \text { hfiber } g b \text {. }
$$

In Coq, this map is defined as

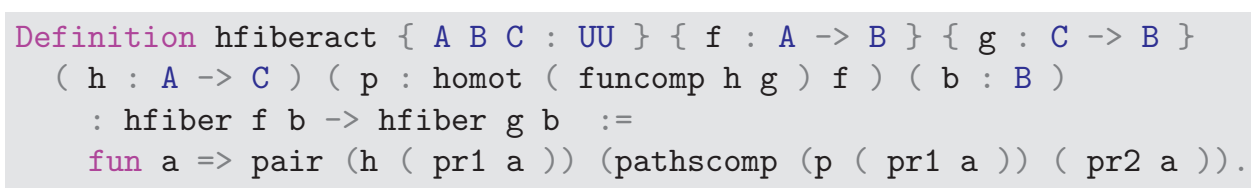

That is, hfiberact $\mathrm{h} \mathrm{p}$ b sends an element

$$
f(a) \stackrel{i}{\longrightarrow} b
$$

of hfiber $f b$ to the point of hfiber $g b$ given by the composite path

$$
g(h(a)) \stackrel{p(a)}{\longrightarrow} f(a) \stackrel{i}{\longrightarrow} b .
$$

As a special case of this construction, when we are given a continuous map $f: A \rightarrow$ $B$ and $g: B \rightarrow C$ together with a point $c$ of $C$, there is an induced map

$$
\text { hfiber }(g \circ f) c \rightarrow \text { hfiber } g c
$$

obtained by applying hfiberact with the identity homotopy $1_{g \circ f}$. In Coq, this is obtained as the following definition:

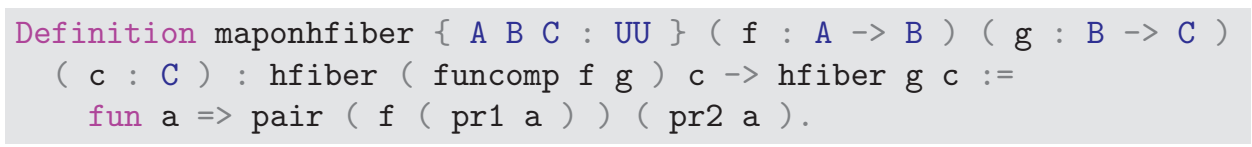

In the case where we have a homotopy retract as in (7.1) above, we obtain, a further map

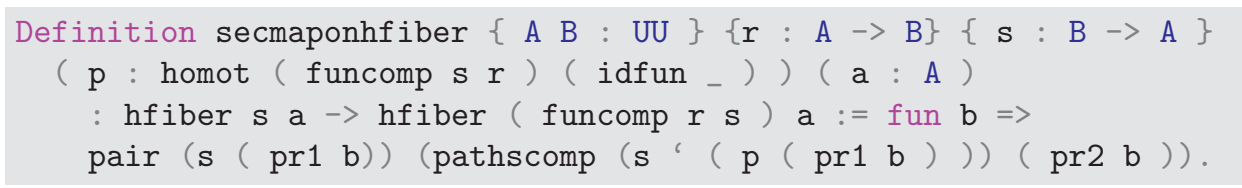


That is, secmaponhfiber sends

$$
s(b) \stackrel{i}{\longrightarrow} a
$$

in hfiber $s$ a to the term in hfiber $(s \circ r) a$ given by the composite path

$$
s(r(s(b))) \stackrel{s^{\prime} p(b)}{\longrightarrow} s(b) \stackrel{i}{\longrightarrow} a .
$$

It turns out that in this situation hfiber $s$ a is a homotopy retract of hfiber $(s \circ r) a$ :

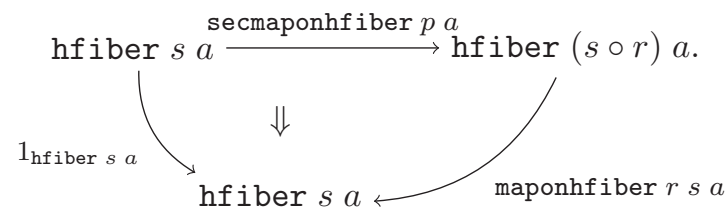

Here, as in (7.1), we indicate the existence of a homotopy in this diagram by a double arrow $\Rightarrow$. In Coq, this fact is captured by the following lemma:

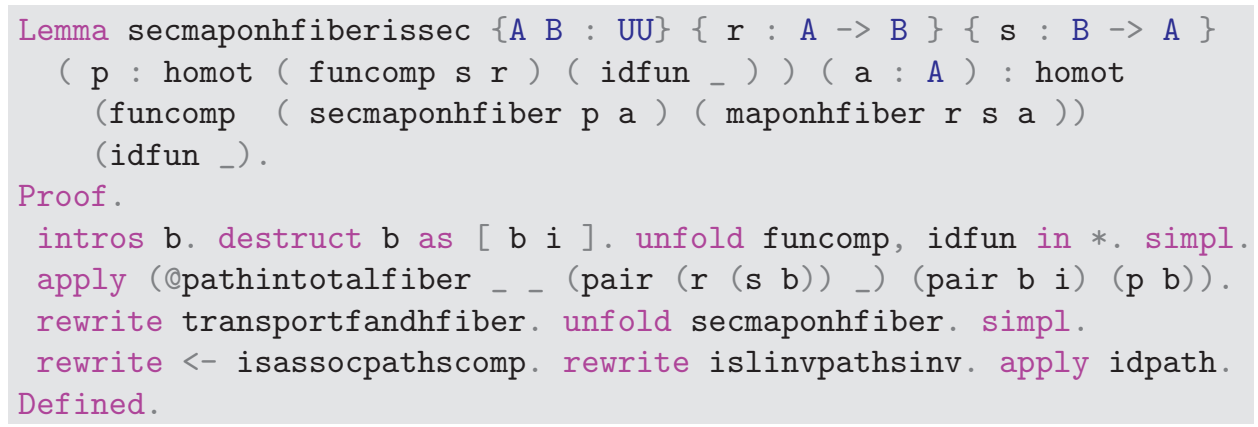

First, after the first application of the simpl tactic, we have simplified the goal and hypotheses to the extent that we are now in the following situation.

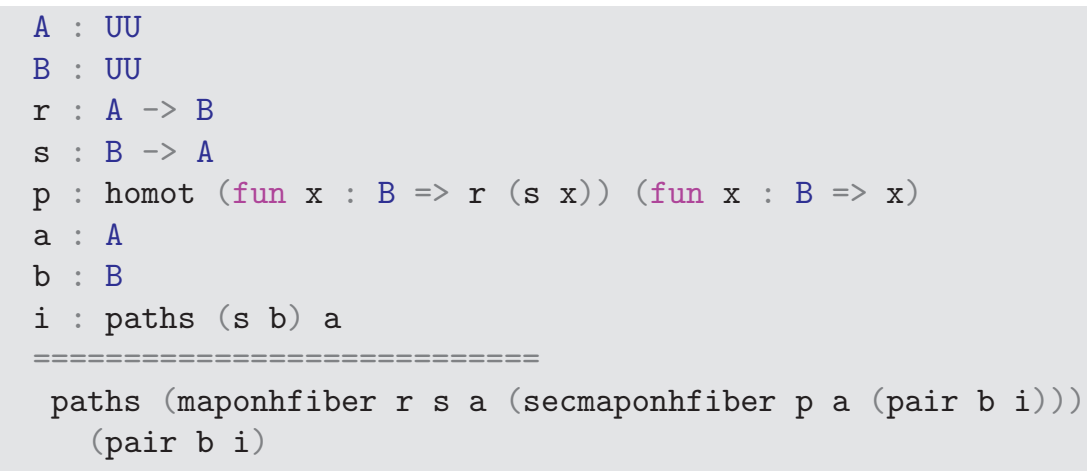

Because we are asked to construct a path in a total space, we will make use of pathintotalfiber. However, in order to help Coq understand exactly what we are trying to do, we must provide some of the implicit arguments of pathintotalfiber explicitly. This is accomplished here using the symbol @. The underscores are the arguments which we leave for Coq to guess, and the other arguments are those we are explicitly providing for Coq. That is, we are telling Coq that in order to achieve the current goal, it suffices to construct using pathintotalfiber a path 
from pair ( $r(\mathrm{~s} b)$ ) - to pair $\mathrm{b}$ i where the path from $r(\mathrm{~s} b)$ to $\mathrm{b}$ we use is $\mathrm{p} \mathrm{b}$. After this, the goal becomes:

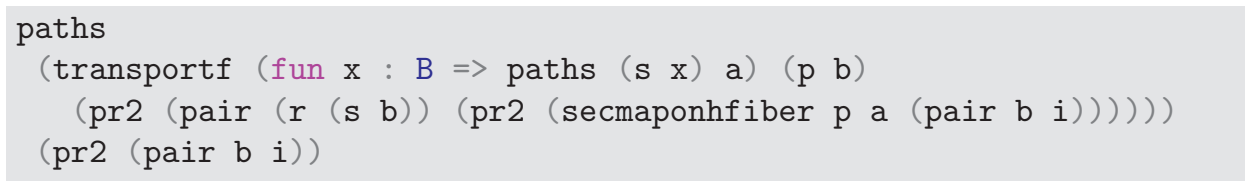

Here, as is often the case, it is convenient to know that the result of applying forward transport can be decomposed in a specific way. In this case it turns out that for $\mathrm{j}$ a path from $\mathrm{v}$ to $\mathrm{w}$ in $\mathrm{B}$ and a path $\mathrm{k}$ : paths $(\mathrm{s} v)$ a,

transportf ( fun $x: B \Rightarrow \operatorname{paths}(s \mathrm{x}$ ) a ) jk

is homotopic (in the sense of path homotopy) to the composite path.

$$
s w \stackrel{\left(s^{6} j\right)^{-1}}{\longrightarrow} s v \stackrel{k}{\longrightarrow} a .
$$

This fact is captured by the lemma transportfandhfiber which has a trivial proof that we omit. Returning to the proof of secmaponhfiberissec, we rewrite using transportfandhfiber and then simplify using unfold and simpl to arrive at the goal

\section{paths (pathscomp (pathsinv ( $\mathrm{s}^{\prime} \mathrm{p}$ b)) (pathscomp ( $\mathrm{s}^{\prime} \mathrm{p}$ b) i)) i}

which is an immediate consequence of associativity of path composition and the fact that pathsinv is an inverse with respect to path composition.

In addition to maps acting via hfiberact, there is also an action of homotopies on homotopy fibers. Namely, a homotopy from a map $f: A \rightarrow B$ to a map $g: A \rightarrow B$ induces, for $b: B$, a map hfiber $f b \rightarrow$ hfiber $g b$ as follows:

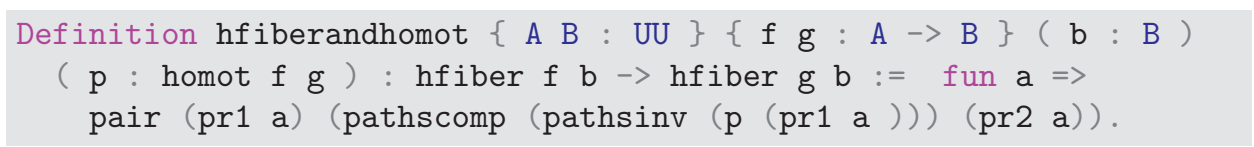

That is to say, this map sends a term

$$
f(a) \longrightarrow b
$$

of hfiber $f b$ to the term

$$
g(a) \stackrel{p_{a}^{-1}}{\longrightarrow} f(a) \longrightarrow
$$

of hfiber $g b$, where $p_{a}$ denotes the component of the homotopy $p$ at $a$.

Similarly, there is a corresponding map

$$
\text { hfiber } g b \rightarrow \text { hfiber } f b
$$

because the homotopy relation is symmetric.

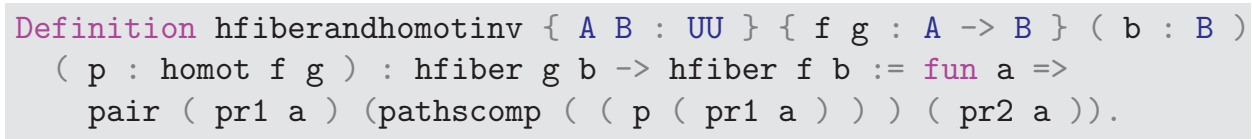

Finally, these two maps constitute a homotopy equivalence of spaces as the following lemma confirms:

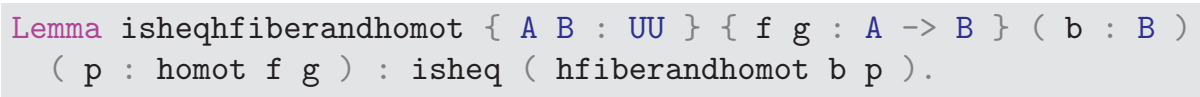


We leave the proof of isheqhfiberandhomot as an exercise for the reader (the proof can also be found in the accompanying Coq file, which is available on the second author's website, http://mawarren.net).

7.3. Weak equivalences. Classically, a map $f: A \rightarrow B$ is a weak equivalence when it induces isomorphisms on homotopy groups. However, we will give a definition, following Voevodsky 78, which makes sense without referring to homotopy groups. In Section 7.4 below we will show that these weak equivalences coincide, for the "nice spaces" we are considering, with the homotopy equivalences (and therefore also with the classical weak equivalences). We start with the definition:

Definition isweq $\{\mathrm{A} B$ : UU $\}$ ( $f: A \rightarrow B):=$

( forall b : B, iscontr ( hfiber $f b$ ) ).

From the "propositions as types" point of view, the weak equivalences correspond to the bijections. The space of weak equivalences from $A$ to $B$ is defined as follows:

Definition weq ( $A B$ : UU ) := total ( fun $f: A \rightarrow B \Rightarrow$ isweq $f$ ).

That is, a typical term of type weq $A B$ is a pair consisting of a map $f: A \rightarrow B$ together with a term of type isweq $f$. The most basic example of a weak equivalence is the identity function idfun A : A $\rightarrow$ A. It is straightforward to construct the required proof that this is a weak equivalence. We denote by isweqidfun $\mathrm{A}$ this term of type isweq ( $\operatorname{idfun~A~),~and~we~then~adopt~the~following~definition:~}$ Definition idweq ( $A$ : UU ) := pair (idfun A ) (isweqidfun A).

That is, idweq $\mathrm{A}$ is the representative of the identity function on $\mathrm{A}$ as a weak equivalence.

Given a map $f$ : A $\rightarrow B$ together with a proof is : isweq $f$ that it is a weak equivalence, if we are given a point $\mathrm{b}: \mathrm{B}$, then there is a corresponding center of contraction in the homotopy fiber of $f$ over $b$ given explicitly by the term pr1 ( is b ). Because it is often convenient to make use the actual term of type A corresponding to this center of contraction, we introduce the following nomenclature:

Definition weqpreimage $\{\mathrm{A} B: \mathrm{UU}\}\{\mathrm{f}: \mathrm{A} \rightarrow \mathrm{B}\}($ is : isweq $\mathrm{f})$
$(\mathrm{b}: \mathrm{B}): \mathrm{A}:=\operatorname{pr} 1(\operatorname{pr} 1($ is $\mathrm{b}))$

That is, weqpreimage is $\mathrm{b}$ is the (homotopically) canonical element in the homotopy fiber of $f$ over b. Similarly, we name the path from $f$ ( weqpreimage is $b$ ) to $\mathrm{b}$ as follows:

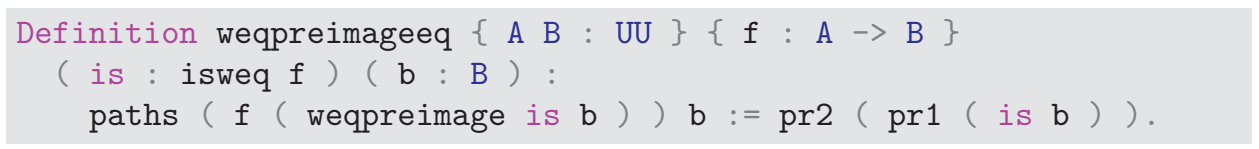

That is, we have

$$
f(\text { weqpreimage is } b) \stackrel{\text { weqpreimageeq is } b}{\longrightarrow} b \text {. }
$$

It is also clear from the definition of weqpreimage that if we are given any point $\mathrm{a}$ : A and path $\mathrm{g}$ from ( $f \mathrm{a}$ ) to $\mathrm{b}$, there exists a path

$$
\text { weqpreimage is } b \stackrel{\text { weqpreimageump } 1 \text { is } b g}{\longrightarrow} a \text {, }
$$


since these both occur in the homotopy fiber over $b$. We leave the construction of the term weqpreimageump 1 to the reader. Finally, the operation of taking the preimage is injective in the sense that if there is a path

$$
\text { weqpreimage is } b \longrightarrow \stackrel{g}{\longrightarrow} \text { weqpreimage is } b^{\prime} \text {, }
$$

then there exists an induced path weqpreimageump2 is $\mathrm{g}$ from $\mathrm{b}$ to $\mathrm{b}$ '.

7.4. Weak equivalences and homotopy equivalences. Given a weak equivalence $f$ : $A \rightarrow B$, we construct a homotopy inverse of $f$ as follows:

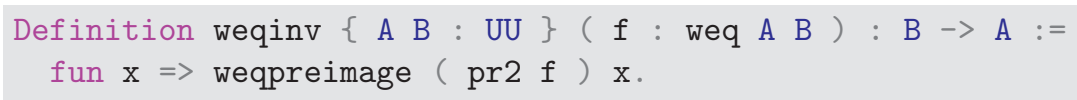

Using the observations from Section 7.3, it is straightforward to prove the following two lemmas:

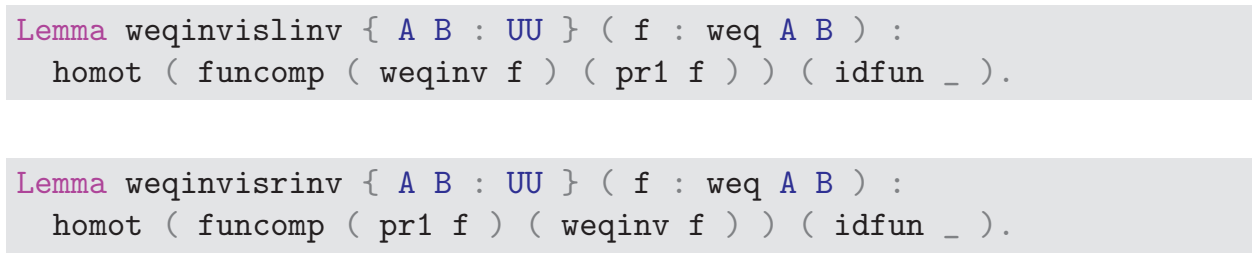

which together constitute the proof of:

Lemma weqtoheq $\{A B: U U\}\{f: A \rightarrow B\}$ (is : isweq $f$ ) : isheq $f$.

Classically, it should be noted that the analogue of weqtoheq only applies in the case of reasonably nice spaces. Interestingly, the proof of the converse of weqtoheq is not entirely trivial. (Categorically, the proof of the converse makes crucial use of the fact that categorical equivalences can be transformed into adjoint equivalences 48.)

The proof of the converse of weqtoheq can be broken up nicely into two lemmas. The first lemma states that, for a homotopy retract as in (7.1) above, if the homotopy fiber of the composite $s \circ r$ over a point $a$ of $A$ is contractible, then the homotopy fiber of $s$ over $a$ is also contractible:

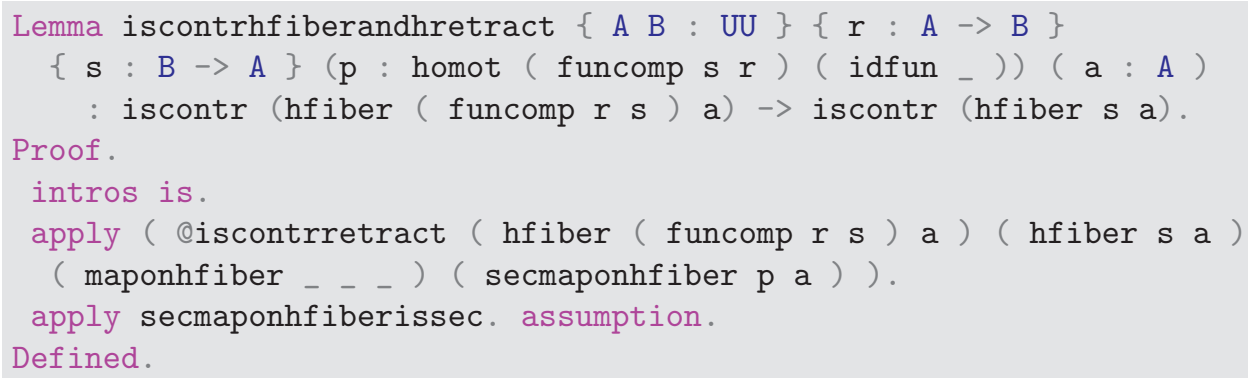

As the Coq code shows, the proof of this fact is an immediate consequence of iscontrretract and secmaponhfiberissec.

The second lemma states that if there is a homotopy from $f: A \rightarrow B$ to $f^{\prime}: A \rightarrow B$ and the homotopy fiber of $f^{\prime}$ over a point $b: B$ is contractible, 
then the homotopy fiber of $f$ over $b$ is also contractible:

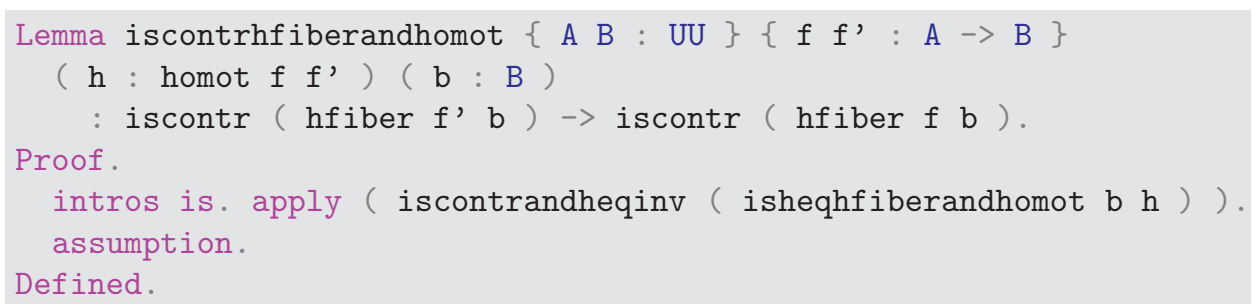

In this case the proof is an immediate consequence of the fact (iscontrandheqinv) that being contractible is preserved by homotopy equivalences together with the fact (isheqhfiberandhomot) that homotopies induce homotopy equivalences of homotopy fibers.

Using these two lemmas we obtain the converse of weqtoheq called, following Voevodsky, the Grad Theorem as follows:

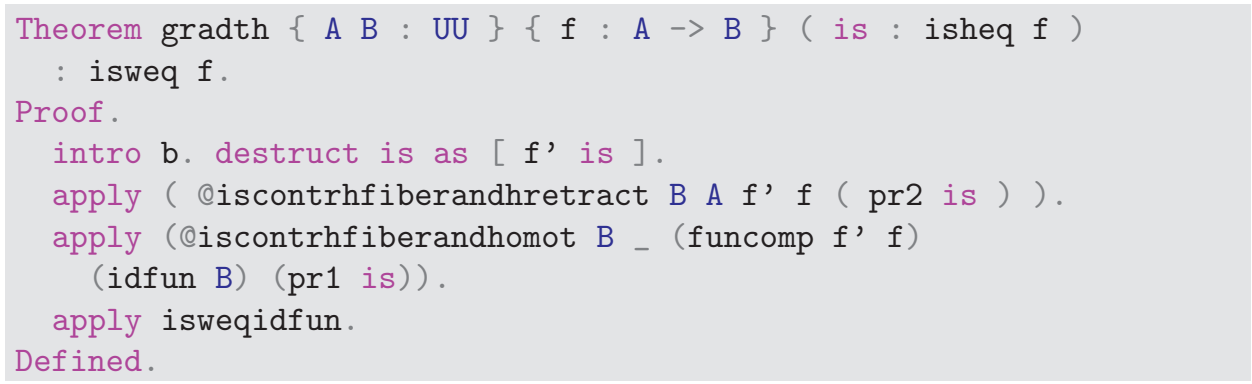

After applying intro b and destruct we find ourselves in the following situation.

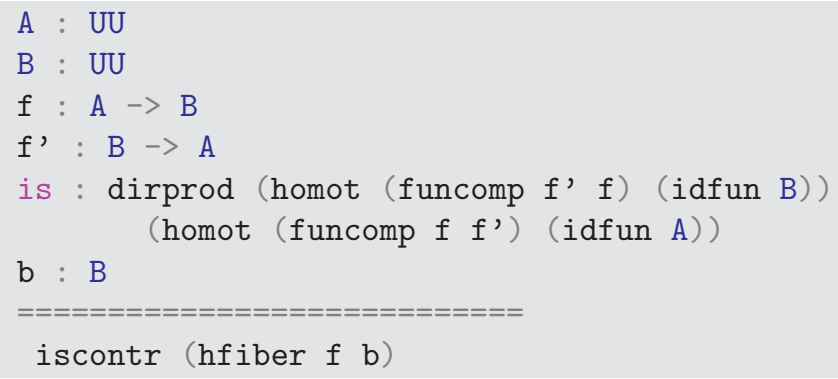

Because $f^{\prime}$ is the homotopy inverse of $f$, by iscontrhfiberandhretract it then suffices to show that the composite $f \circ f^{\prime}$ has a contractible homotopy fiber over $b$. This is the effect of the first apply, after which the Coq output is as follows.

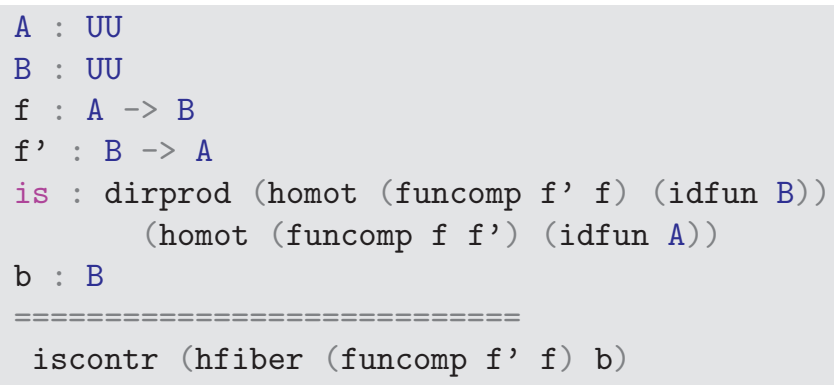


We now observe that there is a homotopy from $f \circ f^{\prime}$ to the identity function on $B$, and therefore after applying iscontrhfiberandhomot, it remains only to prove that the identity on $B$ is a weak equivalences, which is precisely the content of isweqidfun.

\section{The Univalence Axiom and some consequences}

In this section we will describe a number of constructions and results which are more closely related to the univalent approach. Because it would take us too far afield in an introductory paper such as this, we will merely mention a number of the results and display some of the corresponding Coq code. That is to say, the development given here is not self-contained: there are many definitions and lemmas we do not give that would be required in order to obtain all of the results described here.

8.1. The Univalence Axiom. Before giving the explicit statement of the Univalence Axiom, we will first require a map which turns a path in the universe UU into a weak equivalence. For a change of pace, we give a direct definition of this map as follows:

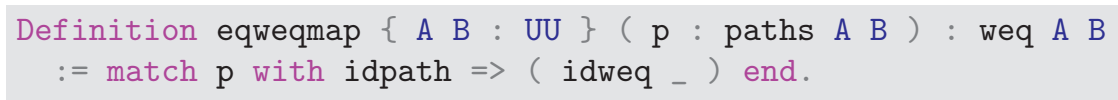

That is, eqweqmap is the map from the path space paths $A$ B to the space weq A B of weak equivalences induced by the operation of sending the identity path on A to the identity weak equivalence idweq $\mathrm{A}$ on $\mathrm{A}$.

We then define the type

Definition isweqeqweqmap := forall A B : UU, isweq (@eqweqmap A B).

The Univalence Axiom then states that there is a term of type isweqeqweqmap. This means that the type of identity paths from $A$ to $B$ in UU is equivalent to the type of weak equivalences from $A$ to $B$. One consequence of this is that the type of weak equivalences from $A$ to $B$ is itself inductively generated in a suitable sense. Indeed, this is the intuition behind a principle which is logically equivalent to the Univalence Axiom and to which we now turn.

8.2. An equivalent formulation of the Univalence Axiom. The idea behind this equivalent form of the Univalence Axiom, which can be seen easily by considering the semantic version of the Univalence Axiom (i.e., what the axiom says in terms of models), states that the type of weak equivalences is inductively generated by the terms of the form idweq A. In particular, they are inductively generated by these terms in the same way that the path space construction paths is inductively generated by the identity paths. Formally, we define the type



In type theoretic notation, weqindelim is the type

$$
\prod_{E:\left(\sum_{X, Y: \mathcal{U}} \text { weq } X Y\right) \rightarrow \mathcal{U}} \prod_{p: \prod_{X: \mathcal{U}}} \prod_{E\left(X,\left(X, 1_{X}\right)\right)} \prod_{X, Y: \mathcal{U}} \prod_{f: \text { weq } X Y} E(X,(Y, f)) .
$$


Intuitively, the space $\sum_{X, Y: \mathcal{U}}$ weq $X Y$ has points given by the following data:

- small spaces $X$ and $Y$;

- weak equivalences $f$ from $X$ to $Y$.

Let us call this space $B$. Then, for a fibration $E \rightarrow B$ over $B$, if there exists a proof $p$ (term) that each fiber $E_{1_{X}}$ over identity weak equivalences $1_{X}$ is inhabited, then a term of type weqindelim $E p$ yields a proof that every fiber $E_{f}$ is inhabited. Thinking of $E$ as a "property" of weak equivalences, this states that in order to prove that a property (definable type theoretically) of weak equivalences holds, it suffices to prove that the property holds of identity weak equivalences. We refer to this as induction on weak equivalences.

In order to prove that if the Univalence Axiom holds, then the induction principle weqindelim also holds, we make use of the following lemma, the proof of which is immediate.

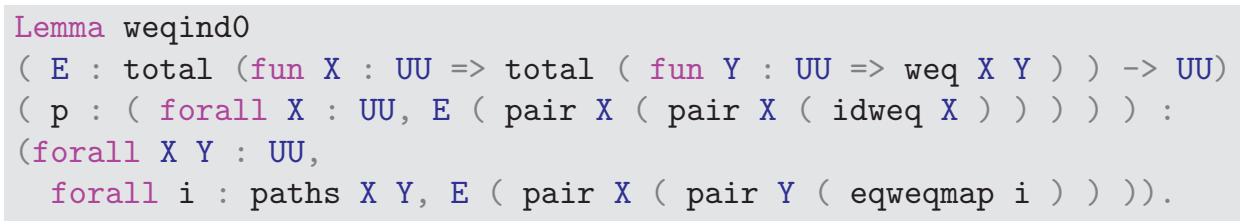

In type theoretic notation, given $E$ and $p$ as in weqindelim above, weqindo gives a term of type

$$
\prod_{X, Y: \mathcal{U}} \prod_{i: \operatorname{Id}_{\mathcal{U}}(X, Y)} E(X,(Y, \text { eqweqmap }(i))) .
$$

I.e., this lemma states that the induction principle in question holds for weak equivalences of the form eqweqmap $(f)$ for $f$ a path between small spaces. Using this, we obtain the following:

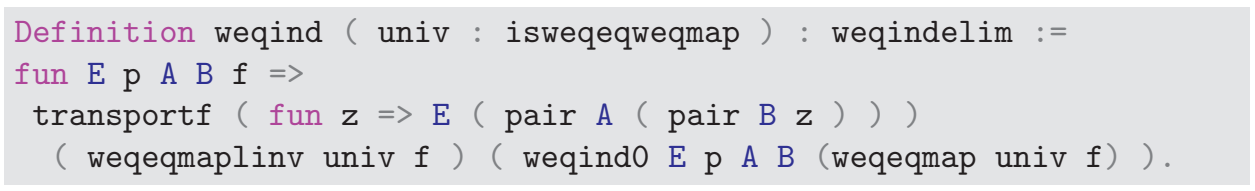

Here we are assuming that the Univalence Axiom holds. I.e., that there is a term univ of type isweqeqweqmap. Then, given all of the data of the induction principle, in order to obtain a proof that the fiber $E_{f}$ is inhabited we observe that, by weqind0, there exists a term $e$ in the

$$
E_{\text {eqweqmap(weqeqmap }(f))},
$$

where weqeqmap is the homotopy inverse of eqweqmap, which exists by the fact that we are assuming the Univalence Axiom. It then suffices to transfer $e$ along the path from eqweqmap(weqeqmap $(f))$ to $f$ (here called weqeqmaplinv).

We note that, when the Univalence Axiom holds, the following computation principle corresponding to weqindelim also holds:

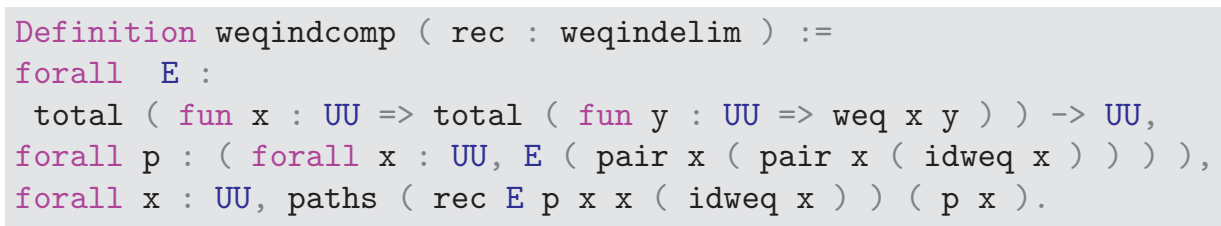


Explicitly, we have the following theorem:

Theorem weqcomp (univ : isweqeqweqmap) : weqindcomp (weqind univ).

The proof of this is slightly involved, and we leave the details to the reader (they can also be found in the companion Coq file for this tutorial). We refer to this computation principle as the computation principle for weak equivalences.

It turns out that the converse implications also hold: in order to prove that the Univalence Axiom holds, it suffices to show that there are terms rec : weqindelim and reccomp : weqindcomp rec. That is, we have the following theorem:

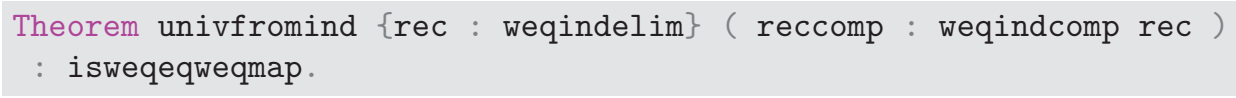

In order to prove this, it suffices, by the Grad Theorem, to prove that eqweqmap has a homotopy inverse. To construct the homotopy inverse, we employ induction on weak equivalences. That is, to construct a map from the space weq A B to the path space paths A B, it suffices to be able to specify the image of an identity weak equivalence. But these are clearly sent to the identity path. The fact that this determines a homotopy inverse is then a consequence of the computation principle for weak equivalences.

8.3. Function extensionality. Henceforth, we assume that the Univalence Axiom holds. That is, we adopt the following:

Axiom univ : isweqeqweqmap.

In Coq, the command Axiom serves to introduce a new global hypothesis. In this case, we assume given a proof univ of the Univalence Axiom. Note that, although a good number do, not all of the facts we prove below require the Univalence Axiom for their proofs. We will begin by briefly summarizing one of Voevodsky's type theoretic results regarding the Univalence Axiom.

One somewhat curious feature of type theory without the Univalence Axiom is that the principle of Function Extensionality is not derivable. Although it can be formulated in a number of ways, the principle of Function Extensionality should be understood as stating that, for continuous maps $f, g: A \rightarrow B$, paths from $f$ to $g$ in the function space $B^{A}$ correspond to homotopies from $f$ to $g$.

Voevodsky [78] showed that Function Extensionality (and a number of closely related principles) is a consequence of the Univalence Axiom. A sketch of the proof written in the usual mathematical style can be found in Gambino 23. We will not describe the proof of Function Extensionality here. We merely mention that we will make use of it in what follows. Explicitly, we make use of a term

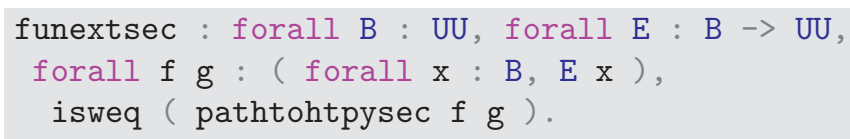

This is a slightly more general form of Function Extensionality and implies the more common version

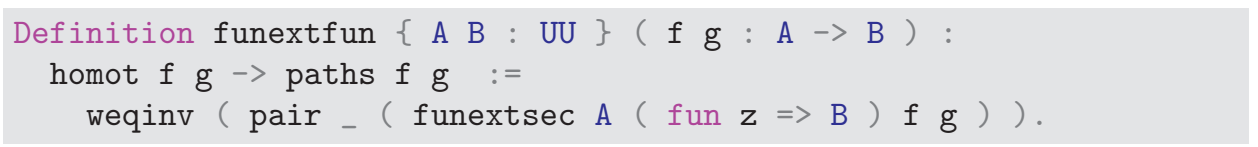


8.4. Closure of h-levels under arbitrary products. We will now describe a number of consequences of the Univalence Axiom concerning h-levels. First, we give the definition of h-levels

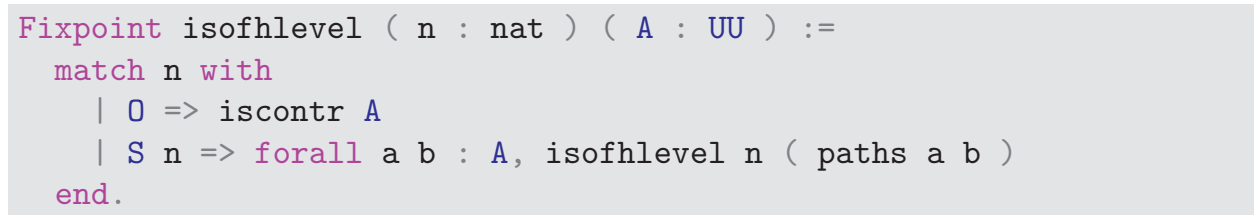

Here the operation Fixpoint tells Coq that we will define a function out of an inductive type (in this case nat) recursively. So, the definition is saying that a type $A$ is of h-level 0 when it is contractible and it is of h-level $(n+1)$ when, for all points $a$ and $b$ of $A$, the path space paths $a b$ is of h-level $n$.

The h-levels satisfy an important property which we will refer to as the impredicativity of h-levels: h-levels are closed under arbitrary products in the sense described below 6 In the base case (for contractible spaces), this is proved as follows:

Lemma impredbase $\{\mathrm{B}: \mathrm{UU}\}(\mathrm{E}: \mathrm{B} \rightarrow \mathrm{UU})$ :

(forall $\mathrm{x}: \mathrm{B}$, iscontr $(\mathrm{E} \mathrm{x})$ ) $\rightarrow$ iscontr (forall $\mathrm{x}: \mathrm{B}, \mathrm{Ex}$ ).

Intuitively, what this says is that given a fibration $E$ over $B$, if every fiber $E_{x}$ is contractible, then the space forall $\mathrm{x}: \mathrm{B}, \mathrm{E} \mathrm{x}$ of sections of the fibration is also contractible. We omit the proof, which is an immediate consequence of funextsec.

The following general principle of impredicativity of h-levels then follows by induction:

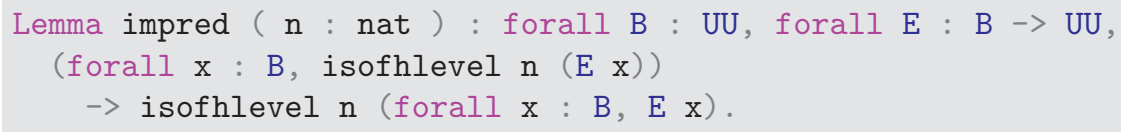

Again, this lemma states that if all fibers $E_{x}$ are of h-level $n$, then so is the space of sections of the fibration.

8.5. The total space and h-levels. Next, we would like to explain the behavior of h-levels when it comes to forming the total space of a fibration. Assume given a fibration $E \rightarrow B$ over $B$. That is, we assume given a term $\mathrm{E}: \mathrm{B} \rightarrow \mathrm{UU}$. Then, for any points $x$ and $y$, there is a weak equivalence between the path space paths $x y$ and the space which consists of pairs $(f, g)$ consisting of paths $f$ from $\pi_{1}(x)$ to $\pi_{1}(y)$ and paths $g$ from $f_{*}\left(\pi_{2}(x)\right)$ to $\pi_{2}(y)$ (see Section [6 above for more on this idea). Using this fact, we obtain the following lemma:

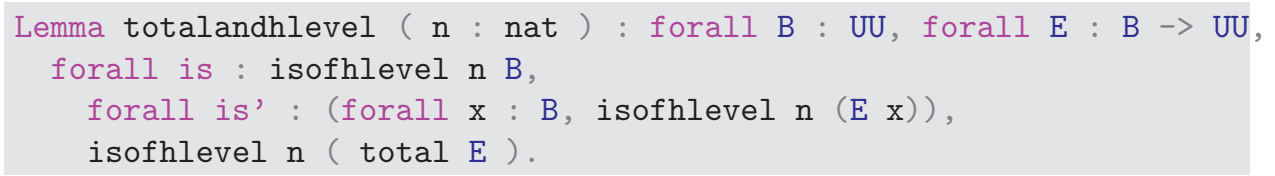

The lemma states that if the base space $B$ and all fibers $E_{x}$ are of h-level $n$, then so is the total space. In the base case $n=0$ this is straightforward. In the induction case, we observe that, for any $x$ and $y$ in the total space,

isofhlevel $\mathrm{n}$ ( paths $\mathrm{x} y$ )

\footnotetext{
${ }^{6}$ There is another notion of impredicativity which is sometimes considered in logic and type theory and should not be confused with this notion.
} 
can be replaced by the Univalence Axiom and the weak equivalence mentioned above by



But the induction hypothesis applies in this case, since we are now dealing with a space of the form total ..., and so we are done.

8.6. The unit type and contractibility. The unit type unit corresponds to the terminal object 1 in the category of spaces under consideration. It is the inductive type with a single generator tt : unit. For any type A there is an induced map tounit $A: A \rightarrow$ unit.

It is a useful fact about contractible spaces that $A$ is contractible if and only if tounit $\mathrm{A}$ is a weak equivalence. We omit the straightforward proof of this equivalence.

One fact about contractible spaces we will require is the fact that if $\mathrm{A}$ is contractible, then so is the type iscontr A of proofs that A is contractible. This is captured by the following lemma:

Lemma iscontrcontr $\{\mathrm{A}$ : $\mathrm{UU}\}$ ( is : iscontr $\mathrm{A}$ ) :

iscontr ( iscontr A).

By the Univalence Axiom and the characterization of contractible spaces mentioned above, in order to prove this lemma it suffices to consider the case where $\mathrm{A}$ is the unit type, which is more or less immediate. This proof reveals one important method for using the Univalence Axiom: to prove something about a space $A$ it suffices, by the Univalence Axiom, to prove the same fact about an easier-to-manage space which is weakly equivalent to $A$.

8.7. Some propositions. We think of types of h-level 1 as being propositions (or truth-values) in the sense familiar to logicians. Following this intuition, we introduce the notation

Notation isaprop:= ( isofhlevel 1 ).

Being a proposition is the same as being proof irrelevant. That is, $P$ is a proposition if and only if, for all terms $p, q: P$, there is a path from $p$ to $q$.

One important consequence of iscontrcontr is the fact that being contractible is itself a proposition:

Lemma isapropiscontr ( A : UU ) : isaprop (iscontr A).

First, note that it is clear that a sufficient condition for being a proposition is being contractible. So, given points $p$ and $q$ of type iscontr A, it suffices to show that the type iscontr A is itself contractible, which is by iscontrcontr.

As a consequence of impredicativity of h-levels together with isapropiscontr, we obtain the following lemma:

Lemma isapropisweq $\{A B: U U\}(f: A \rightarrow B)$

: isaprop ( isweq $f$ ).

So being a weak equivalence is a property of a function. Again using impredicativity of h-levels and isapropisweq, we obtain the following theorem:

Theorem isaxiomunivalence : isaprop (isweqeqweqmap). 
That is, the type of the Univalence Axiom is a proposition, and therefore, assuming that there exists a term univ of this type, the space of such terms is contractible.

Similarly, a straightforward argument shows that, for any space $A$, the type isofhlevel $\mathrm{n} \mathrm{A}$ is a proposition.

8.8. The $\mathbf{h}$-levels of $\mathbf{h}$-universes. We will now consider types of the form

total ( fun $\mathrm{x}$ : $\mathrm{UU} \Rightarrow$ isofhlevel $\mathrm{n} \mathrm{x}$ )

which correspond to the types of all small spaces of a fixed h-level $n$. That is, they are what you might call h-universes. Ultimately, we will compute the h-levels of h-universes. First we will develop some further basic facts about h-levels.

Note that if $A$ is of h-level $n$, then a straightforward argument (using the discussion of h-levels of total spaces above) shows that $A$ is also of h-level $(n+1)$. That is, the h-universes are cumulative. Next, observe that if $B$ is of h-level $(n+1)$, then so is the space of weak equivalences WEq $A B$ for any space $A$ :

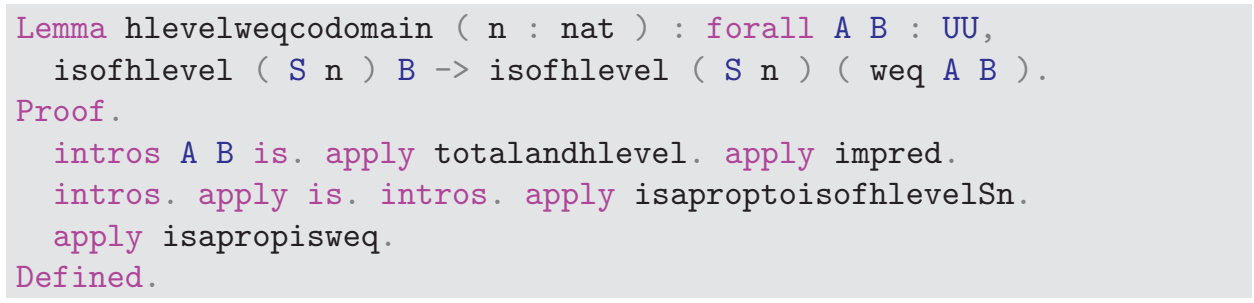

The proof is as follows. It suffices, by totalandhlevel, to prove separately that both the function space A $\rightarrow$ B and the space of proofs that an element $f$ of the function space is a weak equivalence are of h-level $(n+1)$. The former is by impredicativity of h-levels and the latter is by isapropisweq.

Now, for each fixed $n$, there is a version of eqweqmap relativized to the h-universe of type of h-level $n$ :



It turns out that, because isweq $\mathrm{f}$ is a proposition, this map is a weak equivalence:

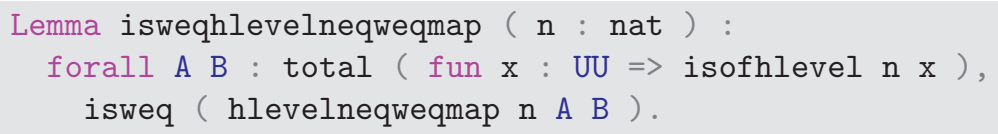

Next, we observe that if both $A$ and $B$ are contractible, then so is the space of weak equivalences from $A$ to $B$ :

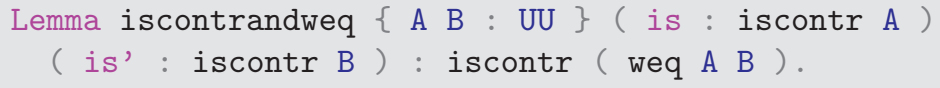

Finally, we observe that the h-universe of types of h-level $n$ itself has h-level $(n+1)$ :

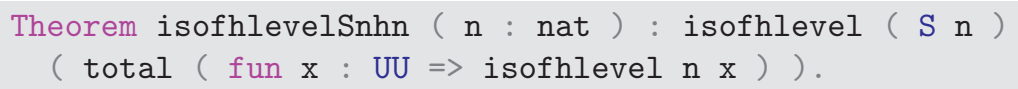

The proof is as follows. First, note that if we are given $A$ and $B$ of type 
then these terms should themselves be thought of as pairs $A=(A, p)$ and $B=(B, q)$ where $p$ is a proof that $A$ is of h-level $n$ and $q$ is a proof that $B$ is of h-level $n$. Nonetheless, there is a weak equivalence between the type paths $(A, p)(B, q)$ and the type WEq $A B$ and as such it suffices to construct a term of type

isofhlevel $\mathrm{n}$ ( weq A B ).

When $n=0$ this is by iscontrandweq and in the case where $n=m+1$ it is by hlevelweqcodomain.

\section{Future Directions}

There are a number of exciting directions in homotopy type theory and univalent foundations which are currently being pursued. We will briefly summarize several of them.

First, there are a number of interesting theoretical questions surrounding the Univalence Axiom which remain open. The most pressing of these questions is the question of the "constructivity" of the Univalence Axiom posed by Voevodsky in 77. Voevodsky conjectured that, in the presence of the Univalence Axiom, for every $t$ : nat, there exists a numeral $n$ : nat and a term of type $\operatorname{Id}_{\text {nat }}(t, n)$. Moreover, it is expected that there exists an algorithm which will return such data when given a term $t$ of type nat. Additionally, there is the question of finding additional models of the Univalence Axiom and characterizing such models (see, e.g., 67]).

Secondly, it remains to be seen how much of modern homotopy theory can be formalized in type theory using either higher-inductive types or some other approach (although a large amount of progress in this direction has already been made; see [1]). Indeed, there is still work to be done to arrive at a complete theoretical understanding of higher-inductive types.

Finally, the formalization of ordinary (set level and higher-level) mathematics in the univalent setting remains to be done. At present, a large amount of mathematics has been formalized by Voevodsky in his Coq library. Additionally, together with Voevodsky, the authors have been working on developing an approach to the theory of integrable systems (using a notion of $p$-adic integrable system as a test case motivated by the real theory [56]) in the univalent setting [57. Ultimately, we hope that it will be possible to formalize large amounts of modern mathematics in the univalent setting, and that doing so will give rise to both new theoretical insights and good numerical algorithms (extracted from code in a proof assistant like Coq) which can be applied to real-world problems by applied mathematicians (cf. [1] for more details on the current progress in this direction).

\section{ACKNOWLEDGMENTS}

We thank Pierre-Louis Curien, Marcelo Fiore, Edward Frenkel, Helmut Hofer, and Vladimir Voevodsky for useful discussions on the topic of this paper. We also thank Chris Kapulkin for several helpful comments on a preliminary draft. Finally, we are grateful to the referees of this article for many valuable comments which have improved the presentation. Syntax-higlighting was produced using the minted LaTeX package. 


\section{ABout The Authors}

Álvaro Pelayo is associate professor at University of California San Diego. He has done research in the areas of symplectic and spectral geometry, semiclassical analysis, and Voevodsky's program. He was a member of IAS during 2010-2013.

Michael A. Warren has carried out research in homotopy type theory and Voevodsky's program, as well as other research in category theory and logic. He was a member of the IAS during 2011-2013.

\section{REFERENCES}

[1] Assorted authors, Univalent foundations of mathematics wiki, 2013, https://uf-ias-2012. wikispaces.com.

[2] Steve Awodey, Type theory and homotopy, Epistemology versus ontology, Log. Epistemol. Unity Sci., vol. 27, Springer, Dordrecht, 2012, pp. 183-201, DOI 10.1007/978-94-007-4435-6_9. MR2962717

[3] Steve Awodey, Pieter Hofstra, and Michael A. Warren, Martin-Löf complexes, Ann. Pure Appl. Logic 164 (2013), no. 10, 928-956, DOI 10.1016/j.apal.2013.05.001. MR3069018

[4] Steve Awodey, Álvaro Pelayo, and Michael A. Warren, Voevodsky's univalence axiom in homotopy type theory, Notices Amer. Math. Soc. 60 (2013), no. 9, 1164-1167, DOI 10.1090/noti1043. MR3113277

[5] Steve Awodey and Michael A. Warren, Homotopy theoretic models of identity types, Math. Proc. Cambridge Philos. Soc. 146 (2009), no. 1, 45-55, DOI 10.1017/S0305004108001783. MR2461866 (2010g:03020)

[6] M. A. Batanin, Monoidal globular categories as a natural environment for the theory of weak n-categories, Adv. Math. 136 (1998), no. 1, 39-103, DOI 10.1006/aima.1998.1724. MR 1623672 (99f:18010)

[7] Hans Joachim Baues, Homotopy types, Handbook of algebraic topology, North-Holland, Amsterdam, 1995, pp. 1-72, DOI 10.1016/B978-044481779-2/50002-X. MR1361886 (97e:55005)

[8] Benno van den Berg and Richard Garner, Types are weak $\omega$-groupoids, Proc. Lond. Math. Soc. (3) 102 (2011), no. 2, 370-394, DOI 10.1112/plms/pdq026. MR2769118(2011m:18010)

[9] Yves Bertot and Pierre Castéran, Interactive theorem proving and program development, Texts in Theoretical Computer Science. An EATCS Series, Springer-Verlag, Berlin, 2004. Coq'Art: the calculus of inductive constructions; With a foreword by Gérard Huet and Christine Paulin-Mohring. MR2229784 (2007i:68001)

[10] Ronald Brown, Topology and groupoids, BookSurge, LLC, Charleston, SC, 2006. Third edition of Elements of modern topology [McGraw-Hill, New York, 1968; MR0227979]; With 1 CDROM (Windows, Macintosh and UNIX). MR2273730

[11] N. G. de Bruijn, The mathematical language AUTOMATH, its usage, and some of its extensions. 1970 Symposium on Automatic Demonstration (Versailles, 1968) pp. 29-61. Lecture Notes in Mathematics, Vol. 125. Springer, Berlin, 1970. MR0267814 (42 \#2716)

[12] Eugenia Cheng, An $\omega$-category with all duals is an $\omega$-groupoid, Appl. Categ. Structures 15 (2007), no. 4, 439-453, DOI 10.1007/s10485-007-9081-8. MR2350215 (2008g:18001)

[13] A. Chlipala, Certified programming with dependent types, to appear. MIT Press. Currently available online at http://adam.chlipala.net/cpdt/, 2012.

[14] Alonzo Church, A set of postulates for the foundation of logic, Ann. of Math. (2) 34 (1933), no. 4, 839-864, DOI 10.2307/1968702. MR.1503136

[15] Alonzo Church, A formulation of the simple theory of types, J. Symbolic Logic 5 (1940), 56-68. MR0001931 (1,321d)

[16] Alonzo Church, The Calculi of Lambda-Conversion, Annals of Mathematics Studies, no. 6, Princeton University Press, Princeton, N. J., 1941. MR.0005274 (3,129b)

[17] Denis-Charles Cisinski, Batanin higher groupoids and homotopy types, Categories in algebra, geometry and mathematical physics, Contemp. Math., vol. 431, Amer. Math. Soc., Providence, RI, 2007, pp. 171-186, DOI 10.1090/conm/431/08272. MR2342828(2008g:55032)

[18] C. Coquand and T. Coquand, Structured type theory, Proceedings of the Workshop on Logical Frameworks and Meta-Languages (LFM'99) (Paris), 1999. 
[19] Thierry Coquand and Gérard Huet, The calculus of constructions, Inform. and Comput. 76 (1988), no. 2-3, 95-120, DOI 10.1016/0890-5401(88)90005-3. MR935892(89j:68096)

[20] H. B. Curry, Functionality in combinatory logic, Proceedings of the National Academy of Sciences 20 (1934), no. 11, 584-590.

[21] Jean Dieudonné, A history of algebraic and differential topology 1900-1960, Modern Birkhäuser Classics, Birkhäuser Boston, Inc., Boston, MA, 2009. Reprint of the 1989 edition [MR0995842]. MR2509981

[22] B. Eckmann and P. J. Hilton, Group-like structures in general categories. I. Multiplications and comultiplications, Math. Ann. 145 (1961/1962), 227-255. MR0136642 (25 \#108)

[23] N. Gambino, The Univalence Axiom and Function Extensionality, Oberwolfach Reports 8 (2011), no. 1, p. 625, Abstracts from the mini-workshop held February 27-March 5, 2011, organized by Steve Awodey, Richard Garner, Per Martin-Löf and Vladimir Voevodsky.

[24] Nicola Gambino and Richard Garner, The identity type weak factorisation system, Theoret. Comput. Sci. 409 (2008), no. 1, 94-109, DOI 10.1016/j.tcs.2008.08.030. MR2469279 (2011d:03011)

[25] R. Gordon, A. J. Power, and Ross Street, Coherence for tricategories, Mem. Amer. Math. Soc. 117 (1995), no. 558, vi+81, DOI 10.1090/memo/0558. MR.1261589 (96j:18002)

[26] A. Grothendieck, Pursuing Stacks, 1983.

[27] Thomas C. Hales, Formal proof, Notices Amer. Math. Soc. 55 (2008), no. 11, 1370-1380. MR2463990(2010h:03012)

[28] Martin Hofmann, On the interpretation of type theory in locally Cartesian closed categories, Computer science logic (Kazimierz, 1994), Lecture Notes in Comput. Sci., vol. 933, Springer, Berlin, 1995, pp. 427-441, DOI 10.1007/BFb0022273. MR 1471244

[29] Martin Hofmann and Thomas Streicher, The groupoid interpretation of type theory, Twentyfive years of constructive type theory (Venice, 1995), Oxford Logic Guides, vol. 36, Oxford Univ. Press, New York, 1998, pp. 83-111. MR1686862

[30] Pieter Hofstra and Michael A. Warren, Combinatorial realizability models of type theory, Ann. Pure Appl. Logic 164 (2013), no. 10, 957-988, DOI 10.1016/j.apal.2013.05.002. MR3069019

[31] W. A. Howard, The formulae-as-types notion of construction, To H. B. Curry: essays on combinatory logic, lambda calculus and formalism, Academic Press, London-New York, 1980, pp. 480-490. MR592816 (82g:03094)

[32] The Univalent Foundations Program Institute for Advanced Study 2013, Homotopy type theory: Univalent foundations of mathematics, 2013, http://homotopytypetheory.org/book.

[33] A. Joyal, I. Moerdijk, and B. Toën, Advanced Course on Simplicial Methods in Higher Categories, Quaderns 45 (2008), no. 2.

[34] Daniel M. Kan, On c. s. s. complexes, Amer. J. Math. 79 (1957), 449-476. MR0090047 $(19,759 \mathrm{e})$

[35] M. M. Kapranov and V. A. Voevodsky, $\infty$-groupoids and homotopy types (English, with French summary), Cahiers Topologie Géom. Différentielle Catég. 32 (1991), no. 1, $29-46$. International Category Theory Meeting (Bangor, 1989 and Cambridge, 1990). MR 1130401 (93c:55006)

[36] C. Kapulkin, P. L. Lumsdaine, and V. Voevodsky, Univalence in simplicial sets, in preparation, on the arXiv as arXiv: 1203.2553, 2012.

[37] U. Kohlenbach, Applied proof theory: proof interpretations and their use in mathematics, Springer Monographs in Mathematics, Springer-Verlag, Berlin, 2008. MR2445721 (2009k:03003)

[38] A. Kolmogoroff, Zur Deutung der intuitionistischen Logik (German), Math. Z. 35 (1932), no. 1, 58-65, DOI 10.1007/BF01186549. MR 1545289

[39] F. William Lawvere, Adjointness in foundations, Repr. Theory Appl. Categ. 16 (2006), 1-16. Reprinted from Dialectica 23 (1969). MR2223032 (2007h:18004)

[40] Tom Leinster, Higher operads, higher categories, London Mathematical Society Lecture Note Series, vol. 298, Cambridge University Press, Cambridge, 2004. MR2094071 (2005h:18030)

[41] D. Licata, A formal proof that the higher fundamental groups are abelian, blog post available at http://homotopytypetheory.org/2011/03/26/higher-fundamental-groups-areabelian/, 2011.

[42] Jean-Louis Loday, Spaces with finitely many nontrivial homotopy groups, J. Pure Appl. Algebra 24 (1982), no. 2, 179-202, DOI 10.1016/0022-4049(82)90014-7. MR651845(83i:55009) 
[43] Jean-Louis Loday and Bruno Vallette, Algebraic operads, Grundlehren der Mathematischen Wissenschaften [Fundamental Principles of Mathematical Sciences], vol. 346, Springer, Heidelberg, 2012. MR2954392

[44] Peter Lefanu Lumsdaine, Weak $\omega$-categories from intensional type theory, Log. Methods Comput. Sci. 6 (2010), no. 3, 3:24, 19, DOI 10.2168/LMCS-6(3:24)2010. MR 2720193 (2011k:03022)

[45] P. L. Lumsdaine, Higher inductive types: a tour of the menagerie, blog post available at http://homotopytypetheory.org/2011/04/24/higher-inductive-types-a-tour-of-themenagerie/, 2011.

[46] P. L. Lumsdaine, Model structures from higher inductive types, preprint, 2011.

[47] Jacob Lurie, Higher topos theory, Annals of Mathematics Studies, vol. 170, Princeton University Press, Princeton, NJ, 2009. MR2522659 (2010j:18001)

[48] Saunders Mac Lane, Categories for the working mathematician, Springer-Verlag, New YorkBerlin, 1971. Graduate Texts in Mathematics, Vol. 5. MR0354798 (50 \#7275)

[49] G. Maltsiniotis, Infini groupoïdes non stricts, d'aprè̀s Grothendieck, preprint, 2007.

[50] P. Martin-Löf, An intuitionistic theory of types: predicative part, Proceedings of the Logic Colloquium (Bristol, July, 1973) (H. E. Rose and J. C. Shepherdson, eds.), Studies in Logic and the Foundations of Mathematics, vol. 80, North-Holland, Amsterdam, 1975, pp. 73-118.

[51] Per Martin-Löf, Constructive mathematics and computer programming, Logic, methodology and philosophy of science, VI (Hannover, 1979), Stud. Logic Found. Math., vol. 104, NorthHolland, Amsterdam, 1982, pp. 153-175, DOI 10.1016/S0049-237X(09)70189-2. MR682410 (85d:03112)

[52] Per Martin-Lö, Intuitionistic type theory, Studies in Proof Theory. Lecture Notes, vol. 1, Bibliopolis, Naples, 1984. Notes by Giovanni Sambin. MR769301 (86j:03005)

[53] Per Martin-Löf, An intuitionistic theory of types, Twenty-five years of constructive type theory (Venice, 1995), 127-172, Oxford Logic Guides, 36, Oxford University Press, New York, 1998. MR 1686864

[54] I. Moerdijk, Fiber bundles and univalence, preprint, available at www.pitt.edu/ krk56/ fiber_bundles_univalence.pdf, 2012.

[55] Ieke Moerdijk and Jan-Alve Svensson, Algebraic classification of equivariant homotopy 2types. I, J. Pure Appl. Algebra 89 (1993), no. 1-2, 187-216, DOI 10.1016/0022-4049(93)90094A. MR 1239560 (94j:55013)

[56] Álvaro Pelayo and San Vũ Ngọc, Symplectic theory of completely integrable Hamiltonian systems, Bull. Amer. Math. Soc. (N.S.) 48 (2011), no. 3, 409-455, DOI 10.1090/S0273-09792011-01338-6. MR2801777 (2012i:37097)

[57] Á. Pelayo, V. Voevodsky, and M. A. Warren, A univalent formalization of the p-adic numbers, to appear in Math. Struct. in Comp. Science, preprint, on the arXiv as arXiv:1302.1207, 2013.

[58] Benjamin C. Pierce, Types and programming languages, MIT Press, Cambridge, MA, 2002. MR.1887075 (2003h:68015)

[59] H. Poincaré, Analysis situs, Journal de l'École Polytechnique (ser 2) 1 (1895), 1-123.

[60] Daniel G. Quillen, Homotopical algebra, Lecture Notes in Mathematics, No. 43, SpringerVerlag, Berlin-New York, 1967. MR0223432 (36 \#6480)

[61] B. Russell, The Principles of Mathematics, 1 ed., Cambridge University Press, Cambridge, 1903.

[62] Dana Scott, Constructive validity, Symposium on Automatic Demonstration (Versailles, 1968), Lecture Notes in Mathematics, Vol. 125, Springer, Berlin, 1970, pp. 237-275. MR0278905 (43 \#4631)

[63] R. A. G. Seely, Locally Cartesian closed categories and type theory, Math. Proc. Cambridge Philos. Soc. 95 (1984), no. 1, 33-48, DOI 10.1017/S0305004100061284. MR727078 (86b:18008)

[64] Jean-Pierre Serre, Homologie singulière des espaces fibrés. Applications (French), Ann. of Math. (2) 54 (1951), 425-505. MR0045386 (13,574g)

[65] M. Shulman, A formal proof that $\pi_{1}\left(s^{1}\right)=z$, blog post available at http:// homotopytypetheory.org/2011/04/29/a-formal-proof-that-pi1s1-is-z/, 2011.

[66] M. Shulman, Homotopy type theory, VI, blog post available at http://golem.ph.utexas. edu/category/2011/04/homotopy_type_theory_vi.html, 2011. 
[67] M. Shulman, The univalence axiom for inverse diagrams, preprint, on the arXiv as arXiv: 1203.3253, 2012.

[68] C. Simpson, Homotopy types of strict 3-groupoids, preprint, on the arXiv as arXiv:math/9810059, 1998.

[69] Carlos Simpson, Computer theorem proving in mathematics, Lett. Math. Phys. 69 (2004), 287-315, DOI 10.1007/s11005-004-0607-9. MR2104448(2005f:03020)

[70] Ross Street, Cosmoi of internal categories, Trans. Amer. Math. Soc. 258 (1980), no. 2, 271318, DOI 10.2307/1998059. MR558176(82a:18007)

[71] T. Streicher, Investigations into intensional type theory, Ph.D. thesis, Habilitation thesis, Ludwig-Maximilians-University Munich, Munich.

[72] W. W. Tait, Constructive reasoning, Logic, Methodology and Philos. Sci. III (Proc. Third Internat. Congr., Amsterdam, 1967), North-Holland, Amsterdam, 1968, pp. 185-199. MR0256877 (41 \#1533)

[73] Zouhair Tamsamani, Sur des notions de n-catégorie et $n$-groupoïde non strictes via des ensembles multi-simpliciaux (French, with English summary), K-Theory 16 (1999), no. 1, 51-99, DOI 10.1023/A:1007747915317. MR1673923 (99m:18007)

[74] The Coq Development Team, The coq proof assistant reference manual, 2012, Version 8.4.

[75] V. Voevodsky, A very short note on the homotopy $\lambda$-calculus, Unpublished note, 2006.

[76] V. Voevodsky, Notes on type systems, Unpublished notes, 2009.

[77] V. Voevodsky, Univalent foundations project, Modified version of an NSF grant application, 2010.

[78] V. Voevodsky, Coq library, available at www.math.ias.edu/ vladimir, 2011.

[79] V. Voevodsky, Experimental library of univalent formalization of mathematics, to appear in Math. Struct. Comp. Sci., preprint available on the arXiv as 1401.0053, 2014.

[80] M. A. Warren, Homotopy Models of Intensional Type Theory, Ph.D. thesis prospectus, Carnegie Mellon University, 2006.

[81] M. A. Warren, Homotopy Theoretic Aspects of Constructive Type Theory, Ph.D. thesis, Carnegie Mellon University, 2008.

[82] Michael A. Warren, The strict $\omega$-groupoid interpretation of type theory, Models, logics, and higher-dimensional categories, CRM Proc. Lecture Notes, vol. 53, Amer. Math. Soc., Providence, RI, 2011, pp. 291-340. MR2867977

Department of Mathematics, University of California San Diego, 9500 Gilman Drive 0112, La Jolla, California 92093-0112

E-mail address: pelayo.alv@gmail.com

Los Angeles, CAlifornia

E-mail address: mwarren@alumni.cmu.edu 